Article

\title{
Design and Implementation of an IoT-Oriented Strain Smart Sensor with Exploratory Capabilities on Energy Harvesting and Magnetorheological Elastomer Transducers
}

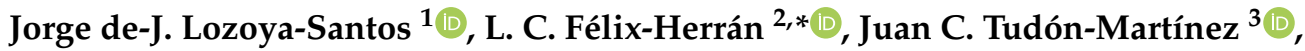 \\ Adriana Vargas-Martinez ${ }^{1}$ (D) and Ricardo A. Ramirez-Mendoza ${ }^{1}$ (D) \\ 1 Tecnologico de Monterrey, School of Engineering and Sciences, Ave. Eugenio Garza Sada 2501, \\ Monterrey 64849, Nuevo Leon, Mexico; jorge.lozoya@tec.mx (J.d.-J.L.-S.); \\ adriana.vargas.mtz@tec.mx (A.V.-M.); ricardo.ramirez@tec.mx (R.A.R.-M.) \\ 2 Tecnologico de Monterrey, School of Engineering and Sciences, Blvd. Enrique Mazón López 965, \\ Hermosillo 83000, Sonora, Mexico \\ 3 Universidad de Monterrey, División de Ingeniería y Tecnología, San Pedro Garza García, Nuevo León 66238, \\ Mexico; juan.tudon@udem.edu \\ * Correspondence: lcfelix@tec.mx
}

Received: 31 March 2020; Accepted: 5 June 2020; Published: 26 June 2020

\begin{abstract}
This work designed and implemented a new low-cost, Internet of Things-oriented, wireless smart sensor prototype to measure mechanical strain. The research effort explores the use of smart materials as transducers, e.g., a magnetorheological elastomer as an electrical-resistance sensor, and a cantilever beam with piezoelectric sensors to harvest energy from vibrations. The study includes subsequent and validated results with a magnetorheological elastomer transducer that contained multiwall carbon nanotubes with iron particles, generated voltage tests from an energy-harvesting system that functions with an array of piezoelectric sensors embedded in a rubber-based cantilever beam, wireless communication to send data from the sensor's central processing unit towards a website that displays and stores the handled data, and an integrated manufactured prototype. Experiments showed that electrical-resistivity variation versus measured strain, and the voltage-generation capability from vibrations have the potential to be employed in smart sensors that could be integrated into commercial solutions to measure strain in automotive and aircraft systems, and civil structures. The reported experiments included cloud-computing capabilities towards a potential Internet of Things application of the smart sensor in the context of monitoring automotive-chassis vibrations and airfoil damage for further analysis and diagnostics, and in general structural-health-monitoring applications.
\end{abstract}

Keywords: smart sensor; magnetorheological elastomer; energy harvesting; piezoelectric; IoT

\section{Introduction}

Damage detection and further diagnosis via structural-health monitoring could be improved with smart sensors that periodically gather, order, and make data available to stakeholders. With the increase of Internet of Things (IoT) applications in the industry, smart sensors are becoming a crucial component, acting not only as transducers, but as an integral solution that incorporates various capabilities into the value chain. Moreover, smart sensors are being applied in several fields, such as healthcare, civil engineering, the automotive industry, and power generation by extending the traditional functions of sensors, i.e., a transducer plus signal conditioning. 
A smart sensor is an electronic device that takes inputs from the physical world, converts the data from one physical domain to another, and contains a signal-conditioning stage. It also processes data through a central processing unit (CPU), and provides information from the gathered data for further decision making. Recently, with the development of new material technologies, smart sensors have also incorporated energy-harvesting, IoT, and cloud-computing capabilities.

Structural-health monitoring (SHM) and further diagnosis are activities that have benefited from the development of smart sensors (including intelligent materials). The integrity of automotive structures, e.g., vehicle chassis, aircraft-wing structures, or structural supports in buildings, is of great economic value, and require measurements of mechanical vibrations and signal conditioning for data processing. Various approaches to monitoring structures have been reported.

Strain gauges are probably the most widely studied and applied transducers to measure strain and mechanical vibrations on structures, but they present some drawbacks. In general terms, strain gauges generate a change in their electrical resistance that is proportional to the applied force that causes an elongation of the gauge. Moreover, the use of strain gauges requires a signal-conditioning stage called the Wheatstone bridge, an intensely studied electrical circuit to measure unknown electrical resistance [1]. Although they have been widely considered, the main disadvantages of conventional strain gauges are their high nonlinearity, temperature compensation, and the need for constant calibration to correctly function [2]. For these reasons, researchers are motivated to replace strain gauges with other materials that do not present these disadvantages.

In the last decade, a type of smart material that has been used as a force-electrical-resistance transducer is the elastomer. Specifically, this work refers to magnetorheological elastomers (MREs) that, rather than cross-linked structures, were applied as force-electrical-signal transducers within rubber molecules due to qualities such as high tensile strength, thermal stability, and increased number of linear structures [3,4]. An MRE is a composite with sensitive magnetic particles that are suspended on a polymeric matrix. MREs belong to a new group of composites that have physical or chemical properties that can be controlled by external stimuli such as magnetic or electric fields, stresses, temperature, moisture, and $\mathrm{pH}$ [5]. Magnetorheological (MR) phenomena in elastomers manifest as a change in the mechanical properties of a viscoelastic material by applying an external magnetic field $[6,7]$.

The availability of required energy in smart sensors is an area of research that has become crucial in recent years. Batteries have been serving as the primary power source to drive these devices. Although batteries have their advantages, they are expensive, take up a significant amount of space, add weight to the sensor, need to be replaced or recharged, and, when discarded, they are highly polluting. In this context, the results of innovative schemes have been focused on harvest energy as an alternative to using batteries.

A promising approach that relies on power extraction from the ambient is known as energy harvesting. This approach is a potentially inexhaustible source of energy for low-power devices. Energy harvesting currently exploits four primary sources of energy-light, electromagnetic radiation, thermal gradients, and motion [8]. The significant drawbacks of energy harvesting are the difficulty in gathering energy and inconstant power generation; hence, different energy-harvesting approaches have been investigated, and piezoelectric materials have yielded favorable results in harvesting energy from mechanical vibrations [9-12]. More specifically to the present study, different approaches to energy harvesting oriented toward structural-health monitoring have been studied [13].

Energy harvesting, which takes advantage of the piezoelectric effect to gather mechanical energy from the environment and to convert it into electrical energy, has been reviewed. Piezoelectric elements have been widely used to collect energy from mechanical vibrations, which make them appropriate for use in ground-vehicle chassis and civil structures, where vibrations within a frequency range impact on the piezoelectric item and generate an output voltage. The most common configuration and design used for piezoelectric-energy harvesting $(\mathrm{PEH})$ employs a cantilever. A piezoelectric cantilever undergoes compression and tension cycles as it is being excited through vibration or cyclic 
forces; however, an important limitation of the cantilever is the limited frequency range in which these devices operate due to their resonant behavior (different beam dimensions); thus, different resonant frequencies and operating bandwidths have been studied [14].

This study presents the design and implementation of a new low-cost prototype of a smart sensor at the macroscopic scale for the measurement of mechanical strain. In addition to measurement and data-processing results, outcomes include an energy-harvesting subsystem with piezoelectric sensors in a cantilever structure and IoT-oriented cloud-computing capabilities through an online dashboard. For the transducer, the ongoing applied approach is to replace strain-gauge arrays with an MRE that contains nanoparticles. Our experimental work was neither nano nor micro, but a macro hands-on endeavor with a prototype built with commercial resources that were not considered to be highly specialized. The use of open connectivity platforms to develop the wireless communication and IoT applications is also highlighted. Moreover, this research includes exhaustive feasibility tests to convert a polymer into a smart material. In this case, different types of nanoparticles were employed to convert a strain signal (the difference between a nominal elongation and that generated when a force is applied to it) into electrical resistance.

The motivation for this work was the monitoring of the chassis of ground vehicles. With information captured from the oscillations and measured strains of these structures, the next step would be to create a network of very efficient (accurate readings, energy harvesting, IoT capabilities) smart sensors to achieve better diagnoses of the state of the structures, including punctual fault location and the remaining lifetime of the structure.

The rest of the article is organized as follows: Section 2 presents related reported work. Section 3 develops the design and implementation methods of each subsystem of the smart sensor, and the employed experiment material, and Section 4 describes the carried-out validation tests. Section 5 presents the preliminary results from the experiment work. Section 6 discusses the results, and Section 7 discusses our conclusions and future work.

\section{Related Work}

Reported efforts about monitoring integrity in automotive and aircraft structures have been reported. One applied method is to track the inner vehicle's chassis, looking for normal deformation of the car's life cycle due to accidents, where a structure of smart sensors detects possible failures on the chassis [15]. Moreover, a low-power-consumption smart sensor for SHM with four piezoelectric transducers was tested to identify impact and damage in automotive and aircraft structures [16]. Another reported outcome of SHM is a virtual test model to detect problems in airfoils [17].

Recently, a magnetoresistive actuator was tested to detect small amounts of damage in aircraft [18]; moreover, a review was carried out on sensing technologies for structural-health monitoring (SHM), and included a case study for a commercial aircraft. Findings showed that the use of smart sensors reduced the time required for aircraft maintenance, and therefore, the cost of maintenance. Contrarily, by increasing the number of sensors, the total weight of the aircraft increased as well as the lifetime cost. Thus, an aim is to create smart sensors that require fewer sensing points to perform structural monitoring [19], such as magnetorheological elastomers (MREs).

Among the different types of MRE, some contain nanoparticles. Due to their suitable characteristics to develop as transducers, this research explored MREs with iron oxide nanopowder and multiwall carbon nanotubes with iron. MR elastomers with iron particles have better mechanical properties and magnetic characteristics compared to those of MREs without the iron microparticles [20]. Furthermore, including carbon nanotubes in the elastomer improves tensile strength, field-dependent storage, loss moduli, and magnetorheological efficiency [21].

A variety of reported results on energy harvesting with piezoelectric elements can be found in the literature. The use of cantilever beams in energy-harvesting systems was widely explored and reviewed [22-26]. Studies focused on the different geometries that the cantilever could have, and, for example, experimental results recorded the generation of a greater voltage with a 
cantilever of triangular geometry against that of others of the rectangular and trapezoidal type [27]. Another research work obtained higher levels of collected energy with a T-shape cantilever [28], whereas another result applied different configurations of the cantilever beam and its impact mechanism to generate electricity [29]. Additionally, there are reported experimental results where a cavity was added to the cantilever beam to increase strain and generated voltage by approximately $75 \%$ compared with those of the option without the cavity [30]. Additional experimental results indicated that the use of multilayered piezoelectric sensors generated more voltage than that of a single-layered structure [31], and showed the use of a cantilever with a coupled pendulum to gather energy from displacements in two degrees of freedom (DOF) [32]. In automotive applications, vibration frequencies are generally very low $(<100 \mathrm{~Hz})$, and for a vehicle chassis and vertical dynamics of the vehicle structure, they are approximately $<15 \mathrm{~Hz}$ [33]. It can be seen from previous works that these frequency bandwidths have not been widely addressed with this kind of mechanism.

Recent smart-sensor applications include the IoT paradigm and taking advantage of cloud computing. Reported SHM results with IoT capabilities were proposed with a tendency to deploy wireless communication as an option to solve wired-sensor technologies [34]. Some approaches have developed remote sensing and a reconfigurable environment such as a wireless-sensor network (WSN) [35], whereas other achievements focused on technology to transfer information among heterogeneous platforms (middleware) within the context of IoT [36]. Moreover, new IoT remote-sensing technologies have been developed. One example is the Xnode wireless platform that has been tested for SHM. On the basis of experimental work, this technology exhibited high reliability and accuracy, in addition to low power consumption. These results identify that this technology has a high potential for SHM with IoT capabilities [37]. Another result is a review on small smart sensors that are able to collect data with IoT technology. The aim of the study was to employ algorithms to infer hidden information [38].

This investigation effort was based on a reported work in progress about smart sensors to measure strain [39], and the developing proof of concept of a smart elastomer for sensing force and vibration [40]. The preliminary experiments that were carried out and the obtained results were extended to obtain broader results that allowed for stronger conclusions. Additionally, the present effort went further into the design and manufacture of a complete prototype, which had not been developed by these previous outcomes, and the energy-harvesting prototype was improved by including more tests, data analysis, and higher levels of generated voltage.

The motivation of this work was to improve reported achievements on the health monitoring of structures through smart sensors. A preliminary result of MRE application on structural monitoring was recently reported [41]. Moreover, another study determined vibration levels and resonance frequencies for vehicles. A Volvo FM12 with air suspension, driven at 30, 50, 70, or $90 \mathrm{~km} / \mathrm{h}$ on three road types, was used for transportation. Vehicle speed was measured with a global-positioning system (GPS), and vibrations were read with two accelerometers placed on the chassis and floor. As a result, four main resonance frequencies were identified for the vertical movement at 1.3, 5.1, 12.6, and $23 \mathrm{~Hz}$. The vibration-exposure values (considering an $8 \mathrm{~h}$ transport period) were $0.61 \pm 0.12 \mathrm{~m} / \mathrm{s}^{2}$, and amplitude of $0.019 \pm 0.0037 \mathrm{~m}$, respectively. According to the results, the energy-harvesting mechanism could be applied in the automotive industry [33]. To the best of the authors' knowledge, there are no reported outcomes regarding energy harvesting with low-cost vibrating cantilevers with embedded piezoelectric sensors that reported generated voltage-peak values greater than $1.0 \mathrm{~V}$ for frequencies of less than $10 \mathrm{~Hz}$. Moreover, a review of the state-of-the-art research did not find reported work about SHM with MRE, IoT characteristics, and energy-harvesting capabilities.

\section{Materials and Methods}

This section presents the design and manufacturing process of the smart sensor, and includes the materials that compose the sensor. Additionally, the tests performed on the finished prototype (transducer + central process unit $(\mathrm{CPU})+$ communication unit + energy harvesting and storage) are 
described to validate and understand their operating characteristics and limitations. The list of used materials (including the computational packages) in the manufacture of the prototype is presented in each subsection that corresponds to the specific subsystem. The schematic of the manufactured prototype is shown in Figure 1.

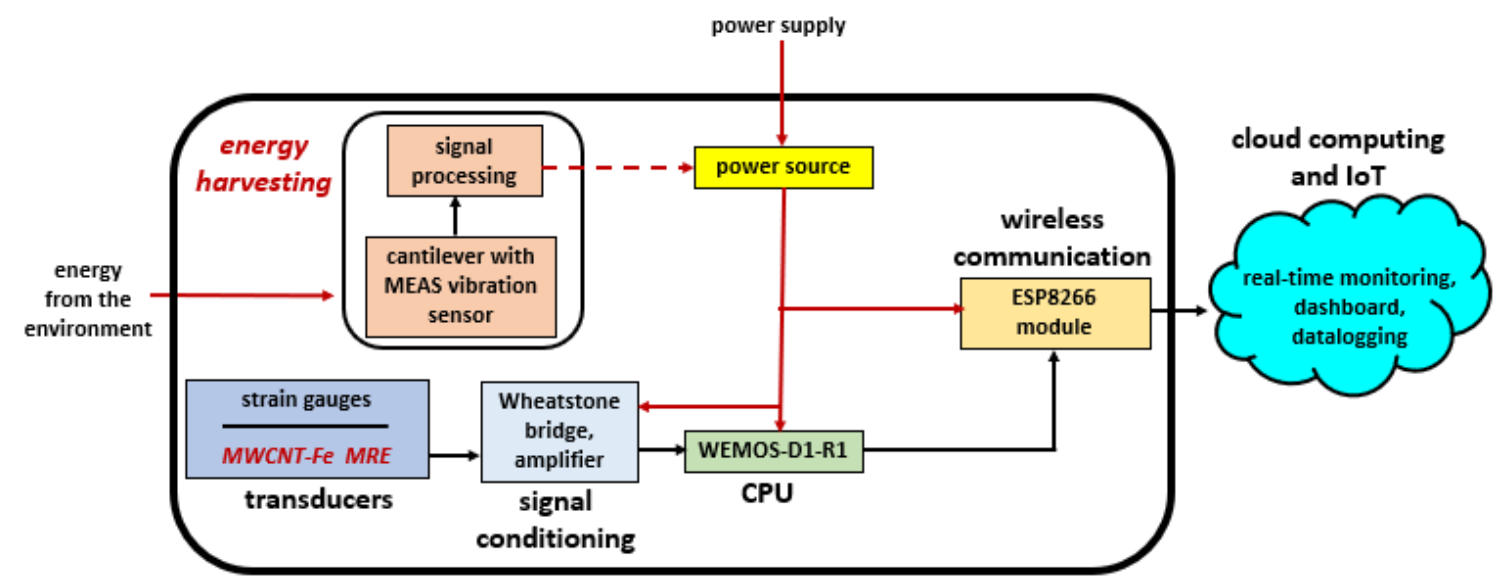

Figure 1. Schematic of the designed and implemented smart sensor. Red text-separately tested subsystems with preliminary results.

\subsection{Design and Manufacturing}

This subsection explains all the work carried out to design and manufacture the smart sensor. The prototype comprised a transducer, a CPU, a wireless module, and a harvesting system.

\subsubsection{Transducer: Magnetorheological Elastomer (MRE)}

A magnetorheological elastomer (MRE) was designed and manufactured to convert strain and vibration measurements into electrical resistance. A general mold was designed using computer-aided-design (CAD) software, and different patterns to measure the strain were tested. Furthermore, this subsection explains, in detail, the manufacturing process used to create three MRE prototypes.

The MRE transducer mold was designed as a 3-layer device. The floor layer contained cavities with a specific pattern based on a strain-gauge rosette. The middle layer was screwed to the floor, and it allowed the samples to be extracted. The top layer was screwed to the middle layer to close the mold. The complete CAD mold is shown in Figure 2.

As part of the research activities, three different designs for the MRE transducer were implemented-a horizontal array with 4 lines, 4 lines in a rosette array with $90^{\circ}$ among lines, and another rosette arrangement with 3 lines and $45^{\circ}$ among them. These designs were modeled in Autodesk Inventor 2018, and printed in 3D with polylactic acid (PLA) material. The array of 4 lines had 4 cavities in the floor layer, describing a pattern of four parallel lines to measure electrical-resistance variations just in one axis. The rosette arrangement with 3 lines of $45^{\circ}$ had a different pattern in the floor layer; it had 3 cavities, one at $0^{\circ}$, another line at $45^{\circ}$, and the third at $90^{\circ}$ to measure electrical-resistance variations in two axes and their components at $45^{\circ}$. The third pattern proposal had two pairs of lines on its floor layer that were placed oppositely and a $90^{\circ}$ rosette strain gauge. The CAD drawings of all the developed patterns are shown in Figure 3.

After the molds were manufactured, to continue the MRE preparation process, preliminary tests of silicon rubber adhesion were performed. Experiments were carried out to verify that a silicone layer could be adhered to another one without mixing. The goal of this experiment was to confirm that both layers were able to merge once they were fully cured. This procedure was applied to the MRE preparation process to combine a layer with a satisfactory percentage of nanoparticles to guarantee an accurate measurement of electrical resistance, with another of lower concentration that served as the 
base material for the transducer. The employed materials for this task were a mold max 30 silicone rubber with its catalyst and the 3D printed molds fabricated from the designs in Figure 3.

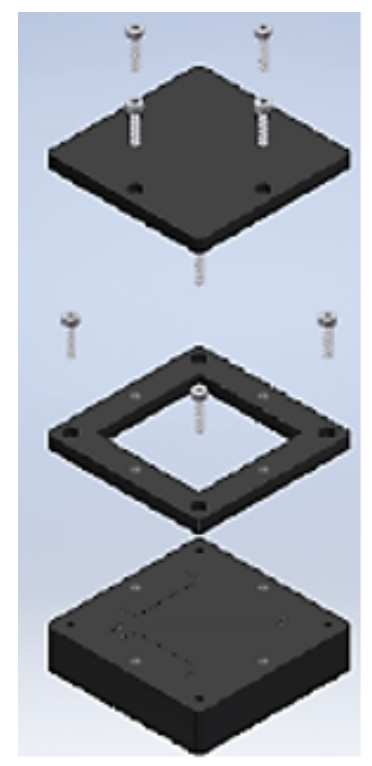

Figure 2. Computer-aided-design (CAD) magnetorheological-elastomer (MRE) mold with 3 layers: floor, middle, and top.

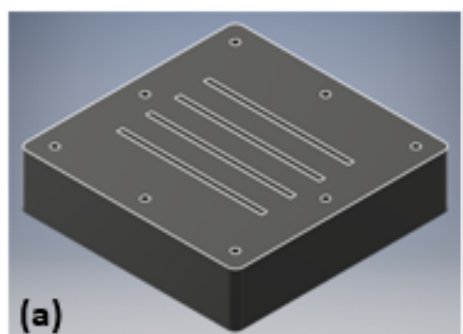

Figure 3. CAD images of three implemented MRE patterns. (a) Horizontal array with 4 lines; (b) 3-line array with $90^{\circ}$ and $45^{\circ}$ between them; (c) 4-line array with $90^{\circ}$ between them. Figures taken from [39].

Silicone rubber and catalyst were applied to generate the MRE. The first experiment used a ratio of 50:1 (98\% silicone rubber and 2\% catalyst). The results were not satisfactory because the low-concentration of catalyst caused the sample to begin the curing process after $20 \mathrm{~h}$. The second and third experiments used a ratio of 10:1 (90\% silicone rubber and 10\% catalyst). The new results from the second and third experiments were acceptable because the mixture started to cure after $2 \mathrm{~h}$, which complied with the reported reaction times during the MRE fabrication process [42]. After a series of trials, the tests were carried out with $90 \%$ silicone rubber and $10 \%$ catalyst, and the results were satisfactory. The layers were successfully merged, and this ratio of rubber to catalyst was used in the subsequent experiments. The manufactured transducers are shown in Figure 4.

The next step was to include nanoparticles in the MRE. An MR elastomer sample composed of two layers was made; the first had a higher concentration of nanoparticles to obtain better electrical conductivity and conduct electrical-resistance measurements in the material, and the second layer had a lower concentration of nanoparticles, with the aim of using it as the base layer.

The first elastomer was fabricated with iron (II and III) oxide nanopowder with a particle size of 50-100 nm, and 97\% trace metal basis. For the preparation of the sample, the first layer had a mass equal to $3 \mathrm{~g}$ consisting of 70\% silicone rubber (ratio 10:1 of silicone rubber to catalyst) and $30 \%$ iron oxide nanoparticles. For the second layer,the mass was equal to $10 \mathrm{~g}$ and it was composed of $90 \%$ silicone rubber (ratio 10:1 of silicone rubber to catalyst), and 10\% iron oxide nanoparticles. 
Electrical-resistance measurements were carried out on the cured sample, but the relation between the applied force and electrical resistance was not evident. The reason for this was that the electrical conductivity of the MRE with iron oxide nanoparticles was too small to exhibit the expected behavior of a potential transducer. To mitigate this, and on the basis of laboratory availability, we used multi-wall carbon nanotubes with iron (MWCNT-Fe). [43].

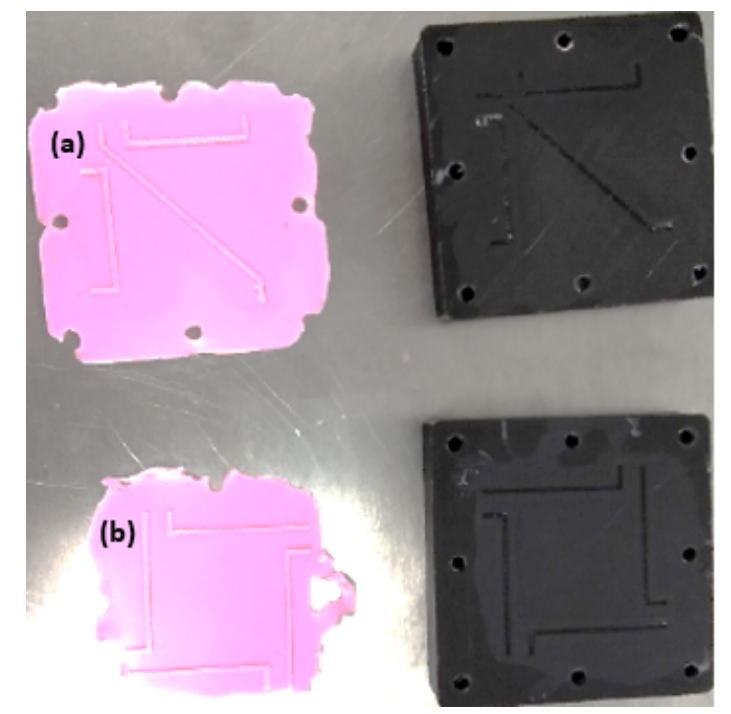

Figure 4. Examples of two generated MR elastomers with $90 \%$ silicone rubber and $10 \%$ catalyst. Depicted patterns are (a) 3-line array with $90^{\circ}$ and $45^{\circ}$ between them, and (b) 4-line array with $90^{\circ}$ between them.

To overcome the aforementioned problem, rubber and catalyst percentages were changed. The first layer consisted of $97 \%$ silicone rubber (ratio 10:1 of silicone rubber to catalyst), and 3\% MWCNT-Fe particles (some trials with a different percentage of particles were carried out before selecting this rubber-nanoparticle percentage ratio). This ratio of silicone rubber to MWCNT-Fe particles was chosen because the second test had satisfactory results-the observed electrical-resistance characteristics were suitable for sensing (i.e., the elastomer exhibited the required elastic behavior). The manufactured 2-layer MR elastomer with nanoparticles is shown in Figure 5.

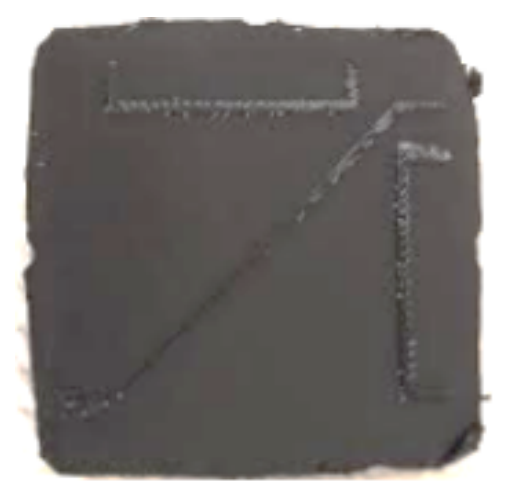

Figure 5. Manufactured 2-layer MR elastomer with nanoparticles.

A graphical way of presenting the set of methods and carried out experiments (planned and executed in sequence) is presented in Figure 6. The image depicts the ordered sequence of laboratory tests. It started with the CAD designs of the molds and continued with the implemented tests for different concentrations of silicone rubber and catalyst. Later, the nanoparticles were added to the correct mixture among layers. In the first trial, the tests included different concentrations of iron (II and III) oxide nano powder but results were not satisfactory, i.e., the relation force vs. electrical resistance 
was poor. Subsequently, the experiments were carried out with MWCNT-Fe and the relation cause (force)-consequence (electrical resistance) was obtained. Ohmmeter tests were applied to the generated samples and based on these measurements, it was validated that the sample with MWCNT-Fe had a better force vs. electrical resistance relation than the iron (II and III) oxide nano powder. The outcomes supported the idea of applying the manufactured MRE as a transducer. With this lately result, three different MRE instances were manufactured (4-lines in parallel, 4-lines in $90^{\circ}$, and 3-lines in $90^{\circ} / 45^{\circ}$ ).

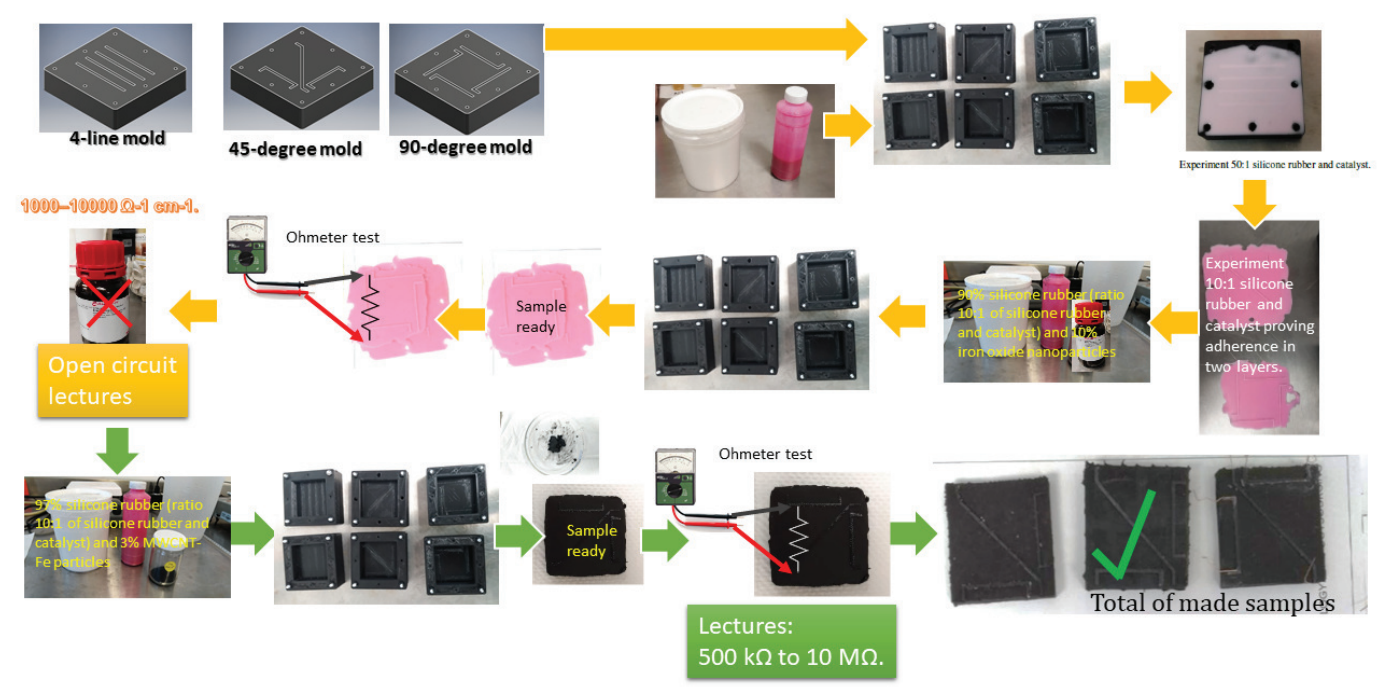

Figure 6. Sequence of experiments during the manufacture of the MRE.

The MRE output is the electrical resistance, which must go through a signal-conditioning stage prior to being processed by the CPU. For this purpose, two required circuits were implemented, a Wheatstone bridge and a signal amplifier with operational amplifiers [1]. Because the signal-conditioning stage for the MRE is still in the development phase, the signal-conditioning circuits were tested as a single subsystem and without the signals generated by the MRE, but with an input signal coming from a strain-gauge array, as explained in Section 5.2. With the obtained outcomes, the signal-conditioning circuits were placed and welded onto a perforated phenolic plate and inside a protective case designed using computer-aided-design (CAD) software.

The protective case consisted of two parts-base and cover-which were attached and secured by holes filled with M3 screws. Moreover, fixed headers were added to the case to connect the conditioning circuit to the CPU module and the strain-gauge terminals. The connection with the CPU module was a 6-pin cable, providing $-5 \mathrm{~V}$, electrical ground, and $+5 \mathrm{~V}$ inputs. The last 3 pins were analog outputs, of which only 1 was used, while the others remained just for symmetry with the CPU case. The case was modelled in 3D with Autodesk Inventor. To manufacture the case, it was printed with polylactic acid (PLA) filament, and all components were stored inside it. The performed phenolic plate was attached with double-sided foam to the bottom of the case, and the data cables were fixed with glue at the sides of the case. The CAD model and the manufactured prototype are shown in Figure 7. 

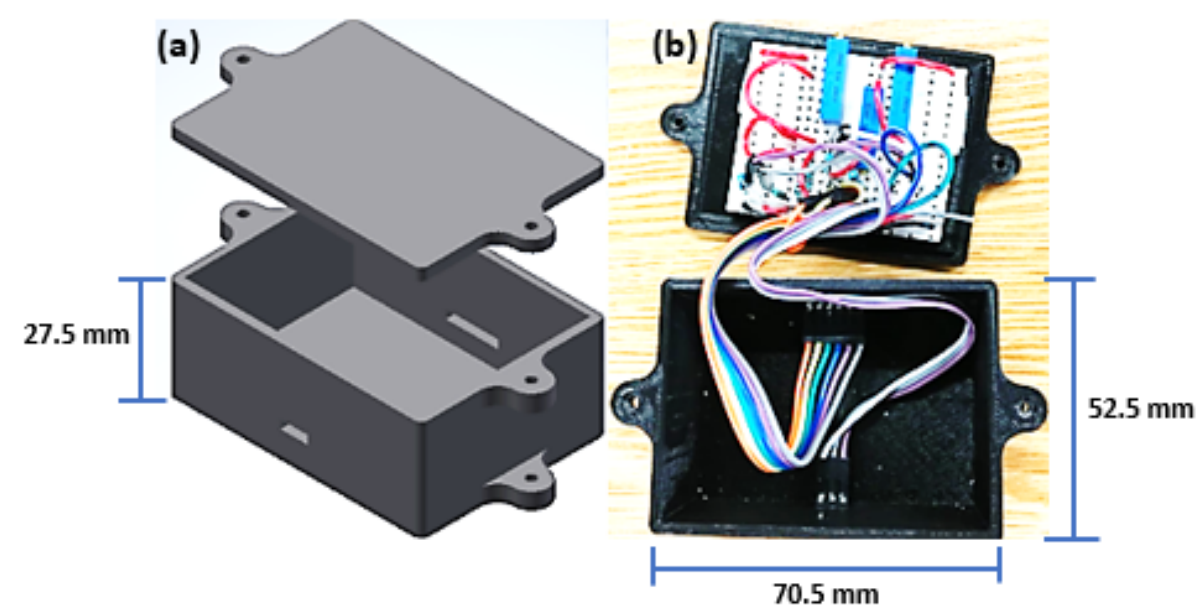

Figure 7. (a) Exploded isometric view of computer-aided design (CAD). (b) Prototype with all the signal-conditioning elements.

\subsubsection{Central Processing Unit (CPU)}

The CPU is the subsystem that administrates the smart sensor. A commercial development board WEMOS-D1-R1 was employed because it met all the sensor requirements, including power supply, input and output pins, microprocessor capabilities, and wireless-communication characteristics.

The Arduino UNO integrated development environment (IDE) was employed to program WEMOS-D1-R1 CPU. This open-source, cross-platform application was compatible with WEMOS-D1-R1, and supported all the necessary features for reading sensor data (programming the 10-bit resolution analog-to-digital converter (ADC), sampling frequencies up to $9600 \mathrm{~Hz}$, and input-pin configuration), and for the communication with the wireless ESP8266 module for data transmission to the cloud.

For safety reasons, the CPU and its connections were placed in protective housing designed with 3D Autodesk Inventor CAD software. The case was divided into 2 parts, base and top, which were joined and secured by screws. Moreover, fixed headers were added to the case to easily interconnect the CPU with the other modules. The connection between the CPU and the energy and harvesting subsystems, which is explained in the following subsection, was a 3-pin cable, providing $-5 \mathrm{~V}$, electrical ground, and $+5 \mathrm{~V}$. For the sensor connection, the case had a 6-pin interface with the previously mentioned electrical signals and 3 analog inputs (A0, A1, and A2). The CAD design and the physical circuits are shown in Figure 8.

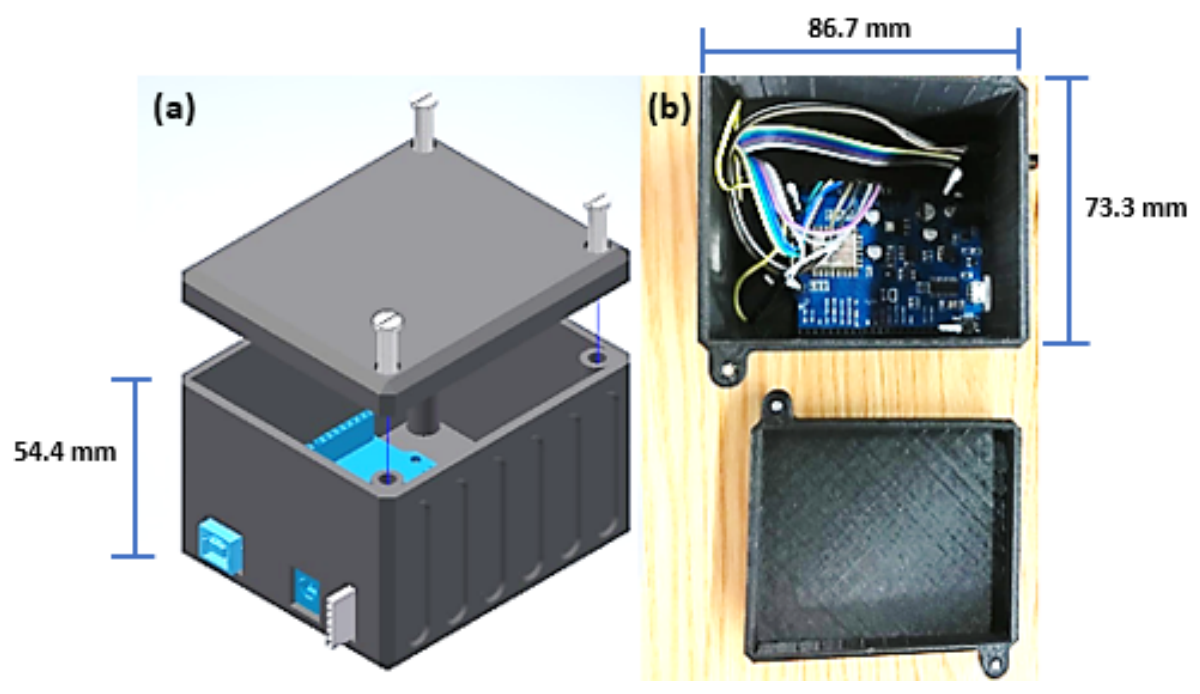

Figure 8. Central processing unit (CPU) subsystem and communication interfaces. (a) Isometric CAD view; (b) manufactured circuit. 
The next task was to manufacture the protective case with PLA material in a 3D printer and to assemble the components. The WEMOS board was secured with 4 screws attached to the bottom face of the case that were locked with metallic nuts. The communication interfaces were set inside the case according to the array of headers established during the case-design phase.

\subsubsection{Wireless-Communication Module and Setup}

Wi-Fi communication was developed to provide Internet capabilities to the smart sensor. Various wireless technologies were reviewed, and the most suitable approach was Wi-Fi technology; therefore, we implemented the ESP8266 Wi-Fi module to allow the CPU to communicate with online services. The ESP8266 Wi-Fi board can be powered with a 5 to $24 \mathrm{~V}$ direct current (DC), and has a current consumption of $170 \mathrm{~mA}$ when transmitting data.

In general terms, communication handling includes some tasks as part of the configuration and data processing. The Wi-Fi keeps the session open; thus, IP address requests are only made once. The Arduino program prepares data to be sent to the data-logging script, which forwards the received information to Google spreadsheets. During these tests, the Wi-Fi achieved a time of $2 \mathrm{~s}$ between each posted lecture. As mentioned before, WEMOS-D1-R1 was programmed with the Arduino IDE. Moreover, the program developed in the Arduino IDE was also required to set the service-set-identifier (SSID) name and its respective password, and to specify the ID of the Google Script to define where to upload the processed data.

A crucial task during this setup was to obtain a fingerprint for the connection session. This fingerprint is generally static for each host, but there are some hosts, such as Google, that deliver fingerprints depending on location services. After the fingerprint was obtained, the program verified that the certificate matched the Google server identification and started sending the collected data from the analog input pin. For the conducted experiment, an open-source terminal was employed (CygWin64).

The next step was to modify the data-logging script. The script for logging data into Google spreadsheets was written in a Google Script language based on JavaScript. There were only two things that were configured in this script, the desired spreadsheet ID, and the selected worksheet inside which the data were to be written.

\subsubsection{Dashboard-Cloud Computing}

Tests were carried out to store some strain readings in a Google spreadsheet. Data were saved in a comma-separated-values (CSV) file, and the gathered data from the sensor were stored and displayed in the spreadsheet. The script read the last saved row that contained information, and then received the information package and provided the needed format to post counter, timestamp, measurement ID, and the raw value in a separate column. At the end of this action, the script increased its counter by 1 so that the correct order was maintained in the spreadsheet.

The IoT approach has information distributed among platforms in order to have offline and online data-analysis capabilities. For this purpose, the dashboard was implemented using the Clic Data website to upload the data log from the Google spreadsheet to the cloud and display it on an online dashboard.

The indicator-panel programming included graphs and numerical variables for the application and a chart. A line chart was selected to present the measured strain as a function of time. Moreover, the dashboard also included columns Strain Value for the section Value, Timestamp for the section Dates, and ID for the section Series. The Click Data website provided all these options within the graph settings, including an update of the displayed data every minute.

To present the results on the dashboard, configuration was carried out within the webpage. The users had to schedule the updating periods or updating cycles for each desired information panel. The shortest time lapse between updates that Clic Data managed was $1 \mathrm{~min}$. The Google spreadsheet needed to be updated along with the dashboard, so the same schedule was set. After displaying 
the link in the browser, it needed to be refreshed by the user to visualize changes on the dashboard, or changes were communicated via email. The server was configured to send an email to the user with the dashboard attached after every update. The summarized data processing from the CPU to the cloud computing and the dashboard followed the sequence in Figure 9.

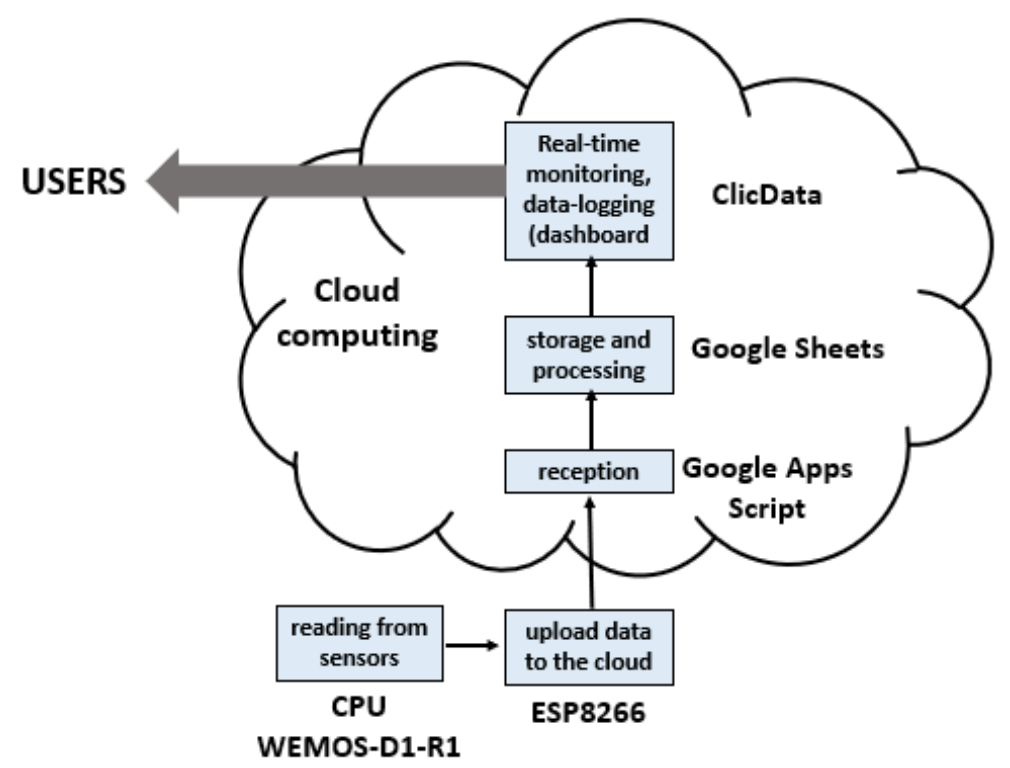

Figure 9. From measurement reading and local processing to online data visualization; dashboard available in the cloud for users.

\subsubsection{Energy-Harvesting System}

The implemented strategy to harvest energy was based on the approach of having piezoelectric sensors attached to a thin steel cantilever beam [44]. The plate was embedded in one side of the structure that held the sensor, and the other end was free to vibrate. This array created vibrations that, in turn, generated voltage through the piezoelectric transducers. Within this baseline design, different types of materials and cantilever structures of a diverse range of lengths were tested to generate energy. All harvesting experiments utilized the flexible piezoelectric vibration sensor by Measurement (MEAS) Specialties, as shown in Figures 10 and 11. Subsequent storage of the harvested energy in this work was not addressed, as we focused on the energy-harvesting system.

A mixture of rubber and catalyst was used to cover the piezoelectric sensor. The combination of materials generated plates that were placed on a protective case. Each hole was designed for a specific screw and its metallic safety nut. This configuration allowed the piezoelectric-sensor and rubber-plate movement by the vibrations to be provided. The rubber plates were connected to the energy-harvesting system that was located below them on the same case.

To create an adequate and efficient way to generate voltage from the piezoelectric element, a rubber cantilever beam was manufactured to increase the vibrations in the piezoelectric sensor. The first step was to create an adequate mold for the rubber structure. The clay mold used for this experiment is shown in Figure 10. After the clay cast for the rubber plate was created, the piezoelectric sensor was positioned to enhance voltage production. The selected position was the edge of the mold, where output cables came out of the mold to connect it to the circuit. 


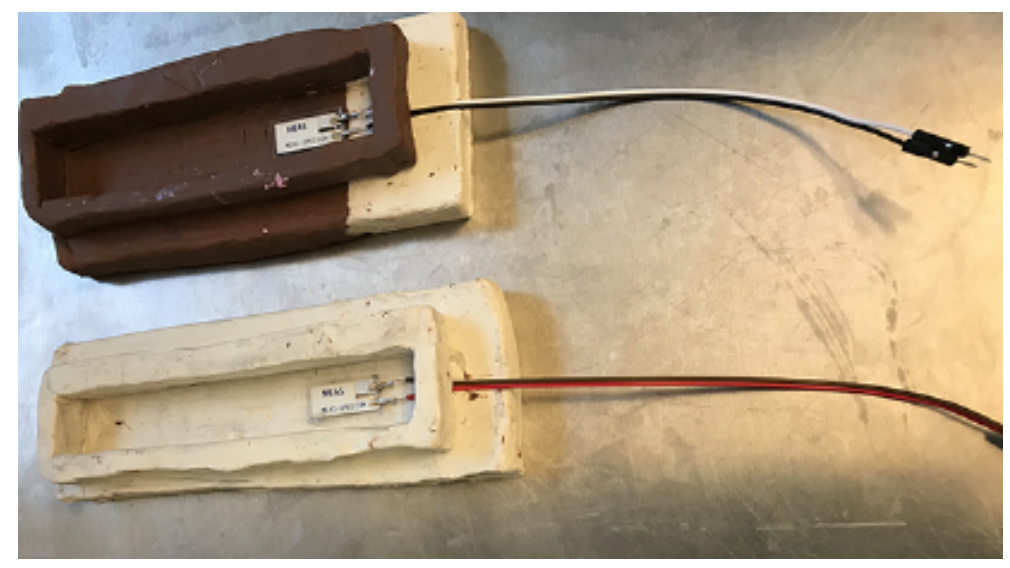

Figure 10. Two implemented clay molds for rubber plates.
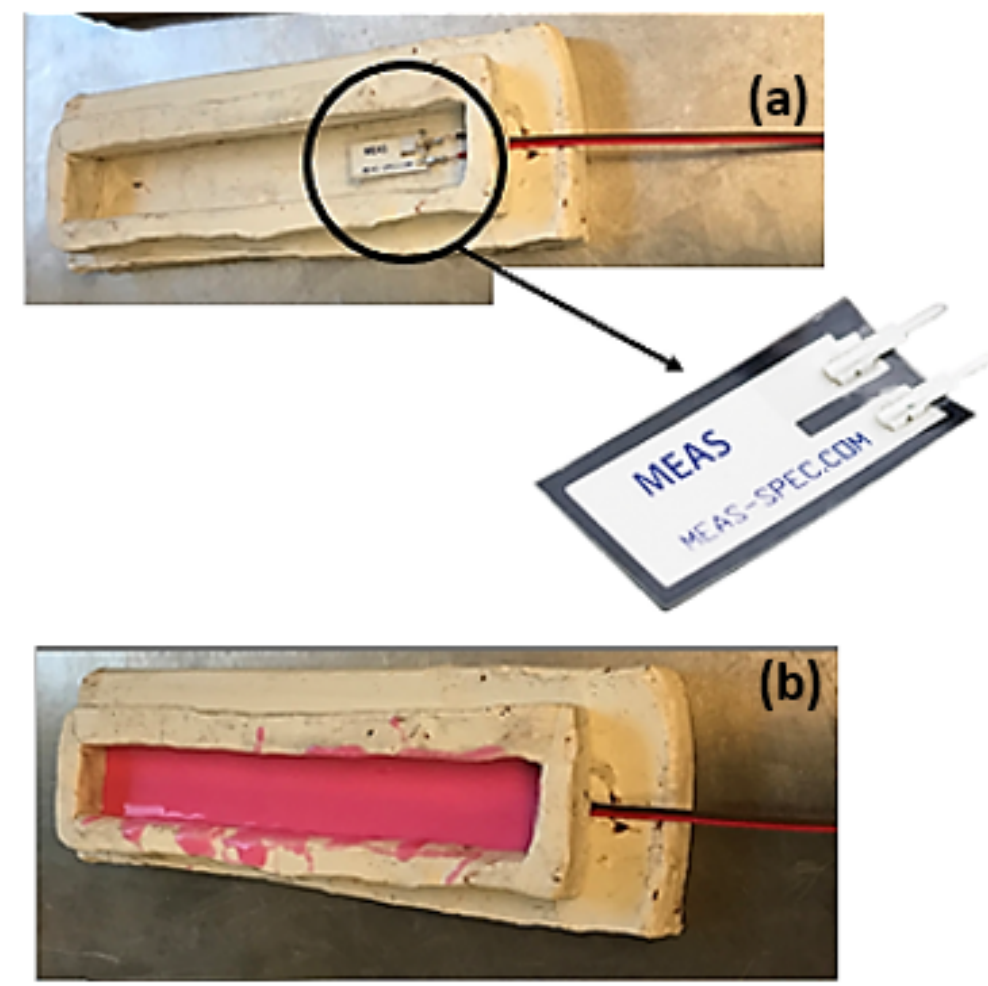

Figure 11. (a) mold filled with rubber-based mixture; (b) MEAS piezovibration sensor inside an empty mold.

A rubber-based solution needs to have the correct amount of each component so that it can be poured into the mold without having excess solution. This is very important due to the restriction posed by the rubber plate's width. If too much solution was poured into the mold, it would result in an increase of the desired width of the rubber plate. After several tests, it was determined that the best components and quantities were white rubber and chemical catalyst, with a composition of $80 \%$ rubber and $20 \%$ catalyst and a total mass of $20 \mathrm{~g}$. The components were poured onto a Petri dish placed on a digital balance to eliminate excess materials. After the masses were verified, elements were mixed with a sterile flat-end laboratory spatula.

Afterwards, the mixture was poured into the mold, maintaining a uniform mixture distribution around the piezoelectric module to ensure that there was an equal amount of rubber around the module to avoid incorrect performance. After the solution was poured, the mix in the mold needed around 2 to $3 \mathrm{~h}$ to fully dry. When this process was completed, the rubber plate was taken out of 
the mold for the testing experiments. The mold when it was full of the mixture and the commercial piezoelectric module is shown in Figure 11.

The harvesting system required a signal-conditioning stage to drive the gathered energy towards the sensor. Currently, proposals for gathering the generated energy from piezoelectric devices have been widely explored and solved, for example, see [45,46]. In this work, for the purpose of analysis, the output of the system, a simple circuit using a $1000 \mu \mathrm{F}$ capacitor and a $1 \mathrm{~N} 4007$ diode conditioned the voltage signal as shown in Figure 12. The output voltage (Vout) was measured between the capacitor terminals that temporarily stored the generated energy.

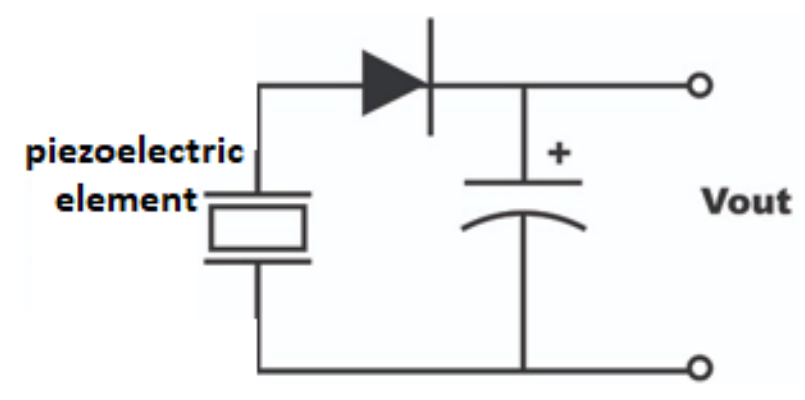

Figure 12. Preliminary circuit to store and deliver harvested energy.

Figure 12 shows that the voltage that was produced by the piezoelectric sensors and temporarily stored in the capacitor passed through the circuit and fed the sensor's batteries. The diode was used to rectify half of the voltage that was going through the circuit. After the sinusoidal signal was halved when it went through the capacitor, it rectified the signal into an average of the signal voltage. This allowed the system to be charged by the voltage exiting the capacitor. To increase the amount of harvested energy, we added two more piezoelectric sensors in parallel [44]. The improved solution is shown in Figure 13.

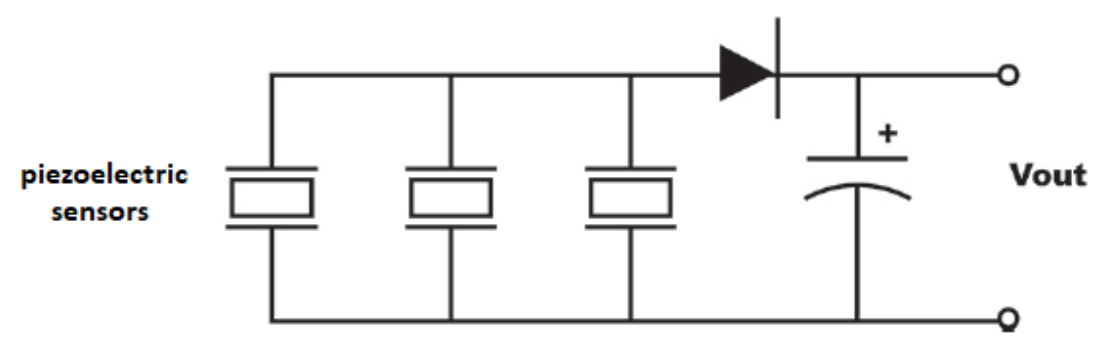

Figure 13. Improved circuit to store and deliver energy.

The next step was to design and implement a protective case for the energy-harvesting subsystem. The container was also designed in CAD software (3D Autodesk Inventor CAD) and 3D-printed with PLA technology. When the CAD was generated, it was ensured that the rubber plates were in contact with the case to allow the piezoelectric sensor and rubber plate to take advantage of the vibrations and thereby increase the quantity of harvested energy. The case was designed with holes on the outside of the box to tie the case to the chassis using belts. The CAD and manufactured solutions are shown in Figure 14. 


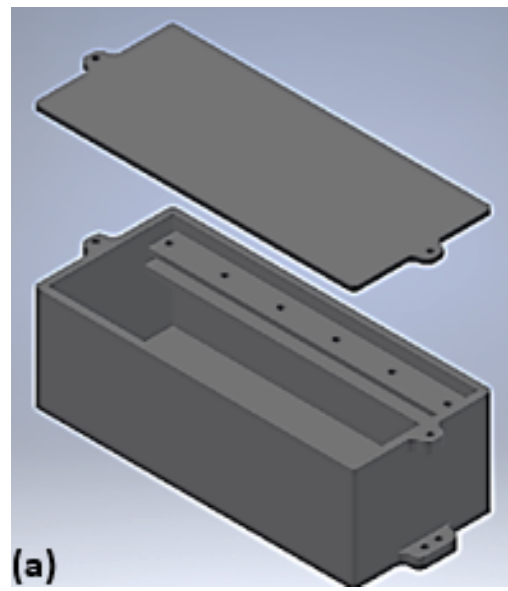

Figure 14. Energy-harvesting subsystem. protective case.

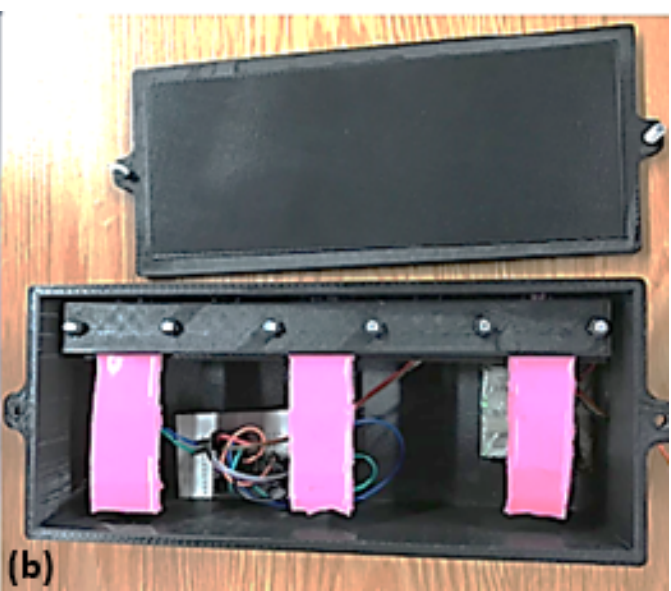

(a) CAD drawing; (b) manufactured subsystem with

\subsubsection{Complete Prototype}

The different subsystems of the smart sensor and the communication aspects between them were investigated separately. The next step was to put all the parts together to create the complete prototype, as shown in Figure 15.

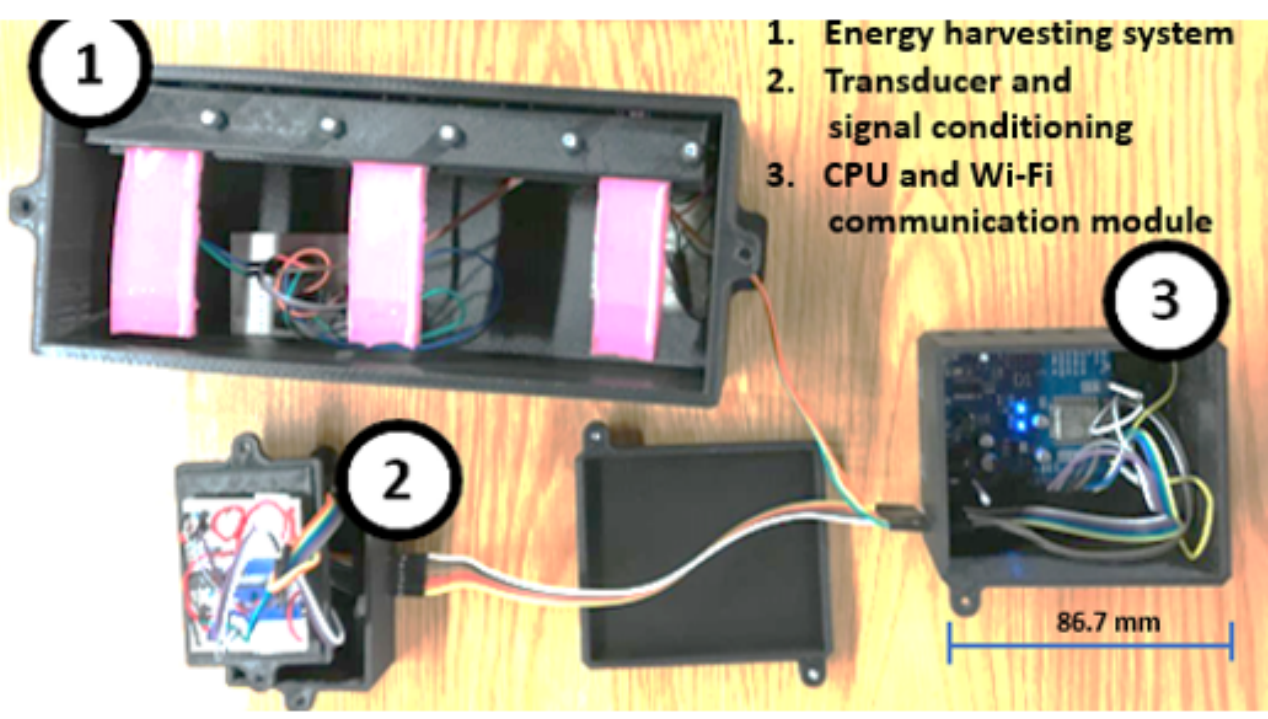

Figure 15. Complete manufactured smart-sensor prototype.

\section{Validation Tests}

To validate the operation of the smart sensor, experiments were separately performed on each subsystem. The following subsections explain the most important aspects regarding the validation tests.

\subsection{Magnetorheological Elastomer}

When the sample was cured, some electrical-resistance tests were carried out on the MRE sample to verify if MWCNT-Fe particles increased the electrical conductivity. Electrical-resistance values within a range from $500 \mathrm{k} \Omega$ to $10 \mathrm{M} \Omega$ were measured during the experiment; the results supported the use of multiwall carbon nanotubes with iron (MWCNT-Fe) instead of iron (II and III) oxide nanopowder. This validation work considered some tension-compression tests with MRE reported in the literature [47-49]. 
An open-force-tester machine was used to measure the strain for MRE. The LYNX testing software from the machine measured the total applied force and the length by which the MRE could be stretched before becoming loose. For the setup, the machine was set at an initial position; then, the MRE was fixed into the open force tester-into the bottom and top sections of the center base of the tester-having been attached to measure resistance. The center base was where the test subjects were placed, with detachable lower and upper bases in order to adjust to the size of what was being tested. The bottom base was static, while the upper base was the one that moved upward and downward; for tension, it moved up. At the start, the LYNX software captured the force that was being applied to the MRE while it was being stretched. At the same time, the program measured the elongating distance, and the multimeter read the electrical resistance. Twelve MRE samples were tested under tension considering three different designs for each MRE (each with a different distribution of nanoparticles) to find which design exhibited the highest resistance measurement. With the gathered data, a graph of applied force vs electrical resistance was obtained. Tensile testing efforts included two replicas of the same testing conditions to obtain more reliable results. The experimental setup for the tensile tests is shown in Figure 16.

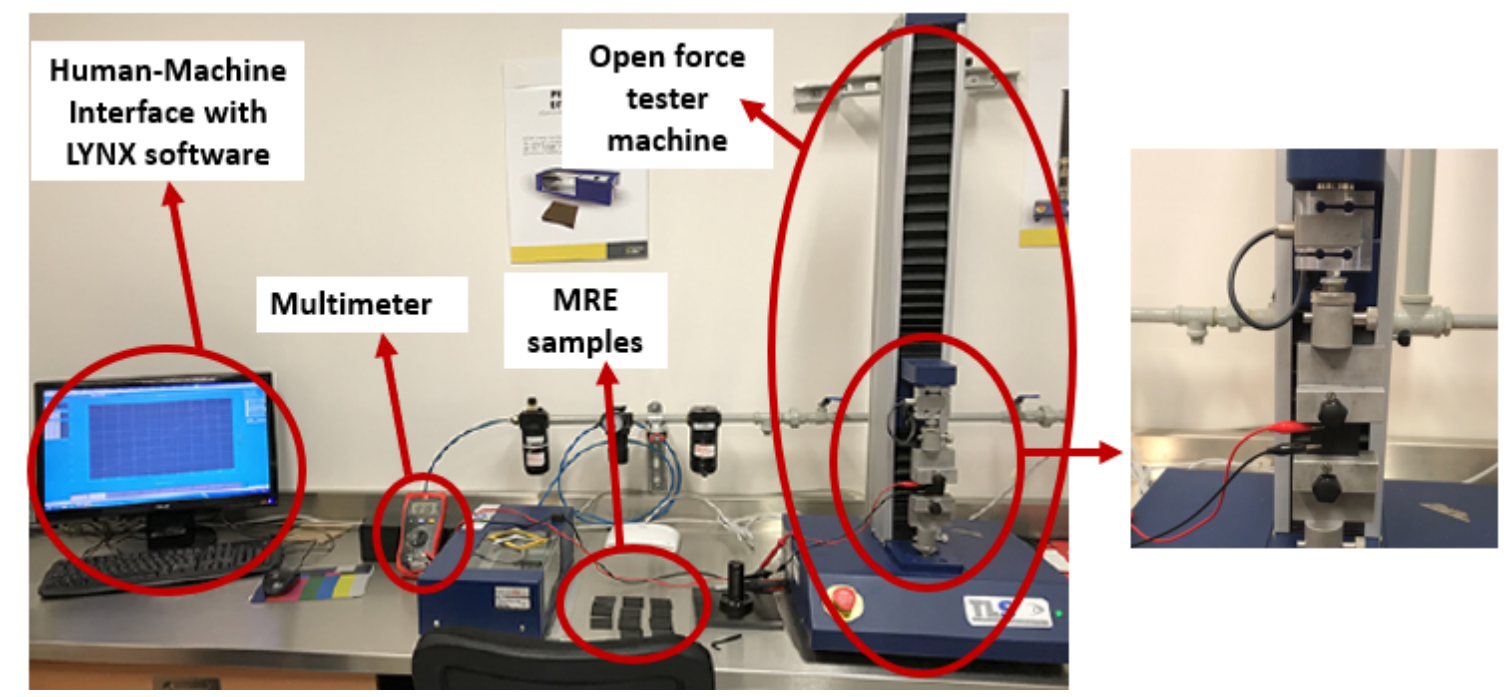

Figure 16. Laboratory setup for MRE tensile tests.

Compression tests were also performed to validate the behavior of the electrical resistance of the MRE samples. In the laboratory, a hydraulic jack was used to apply force to a steel surface where the MRE samples were placed, resulting in their compression. The force applied by the hydraulic jack was measured with a dynamometer, and to register the measurements of electrical resistance. National Instruments (NI) Educational Laboratory Virtual Instrumentation Suite (NI ELVIS) II was used with its multimeter function to measure electrical resistance. Measurements were taken and displayed in real time through a human-machine interface (HMI) in NI LabView. As performed with the tensile testing, two replicas for the same testing conditions were registered to obtain more reliable results.

The gathered data were stored in an Excel file for additional processing. For the experiments, the three types of MRE samples in Figure 3 were tested. Each sample was fixed on a steel surface, and two crocodile clips connected the MRE sample to the data-acquisition (DAQ) system. With this setup, a hydraulic jack was employed to apply the force to compress the MRE sample. The NI ELVIS II took measurements of electrical resistance, and the HMI displayed the data in a waveform chart. When the test was finished, data were stored in an Excel spreadsheet. For the experiments, the applied force was in the range of 0 to $2250 \mathrm{~N}$ in steps of $250 \mathrm{~N}$, with a total test duration of approximately $80 \mathrm{~s}$. The compression-test equipment, including data acquisition, is shown in Figure 17. 


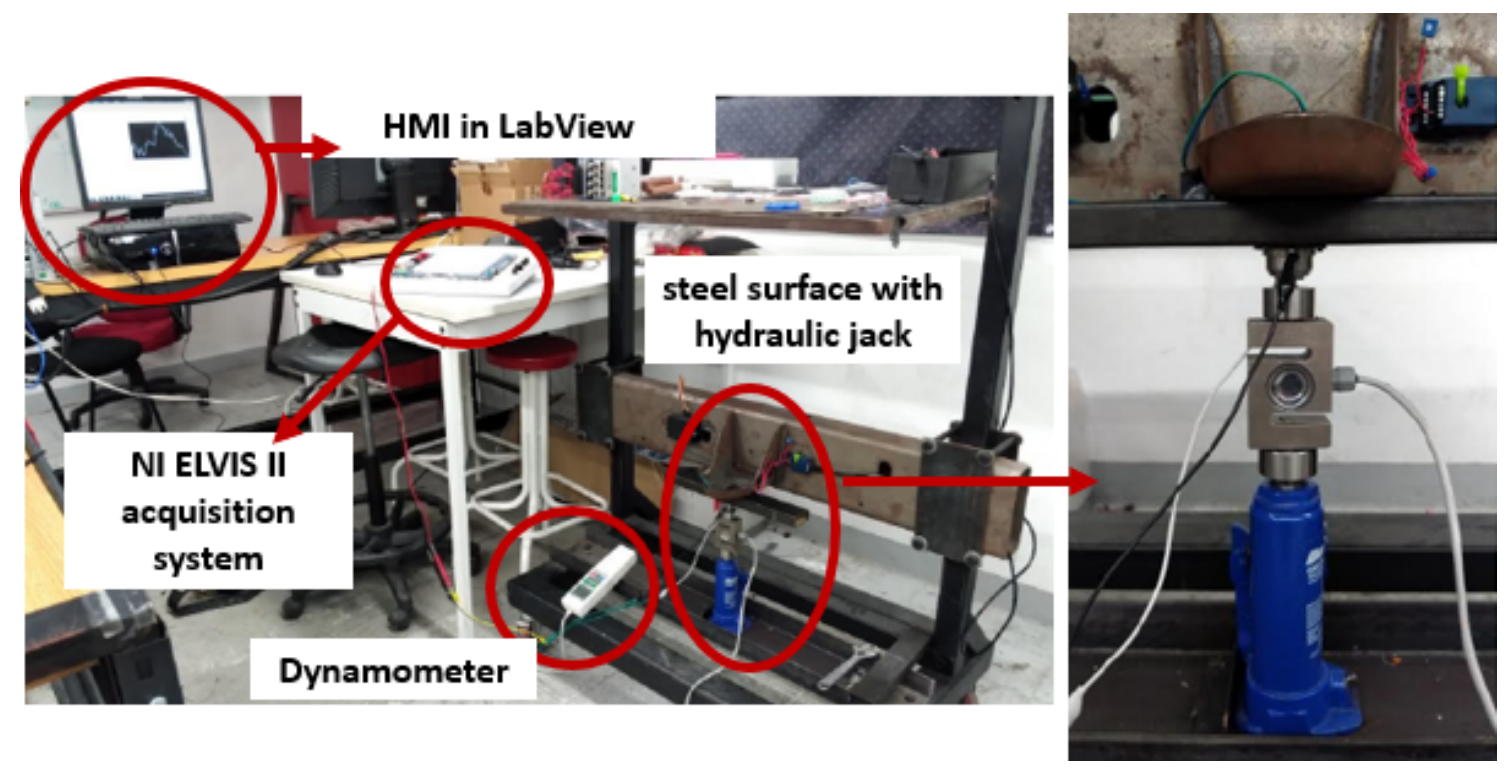

Figure 17. Laboratory setup for compression tests. Figures modified from [40].

\subsection{Energy Harvesting-Preliminary Tests}

To determine the most efficient way for the rubber plates to generate a voltage, certain tests were performed in the lab. For this analysis, the employed machine was able to vary the frequency of the applied input.

For the experiments, the rubber plate had a length of $8 \mathrm{~cm}$, and the machine was set to vibrate in a frequency range from 4 to $9 \mathrm{~Hz}$ (in steps of $1 \mathrm{~Hz}$ ), spending $35 \mathrm{~s}$ in each frequency attempt. The purpose of using several frequencies was to find a frequency that would generate the highest amount. For each sample, the test was done three times to avoid relying on just one test. After the three tests were completed, the length of the rubber plate was reduced to $7.5 \mathrm{~cm}$. Different lengths and frequencies were tested with the aim of finding the combination that harvested the highest amount of energy. For each frequency range and the three tests per length, the length of the rubber plate was decreased to $5.5 \mathrm{~cm}$ (in steps of $0.5 \mathrm{~cm}$ ).

Figure 18 shows that the rubber plate was placed beneath a metal structure and on the edge of the vibration machine to enable the rubber plate to move freely. The circuit was connected to a DAQ system and a human-machine interface (HMI) developed using the National Instruments LabView software. The HMI had the option to export the data to an Excel spreadsheet. Further analyses in Excel allowed for the length and frequency combination that produced the highest voltage to be determined. 


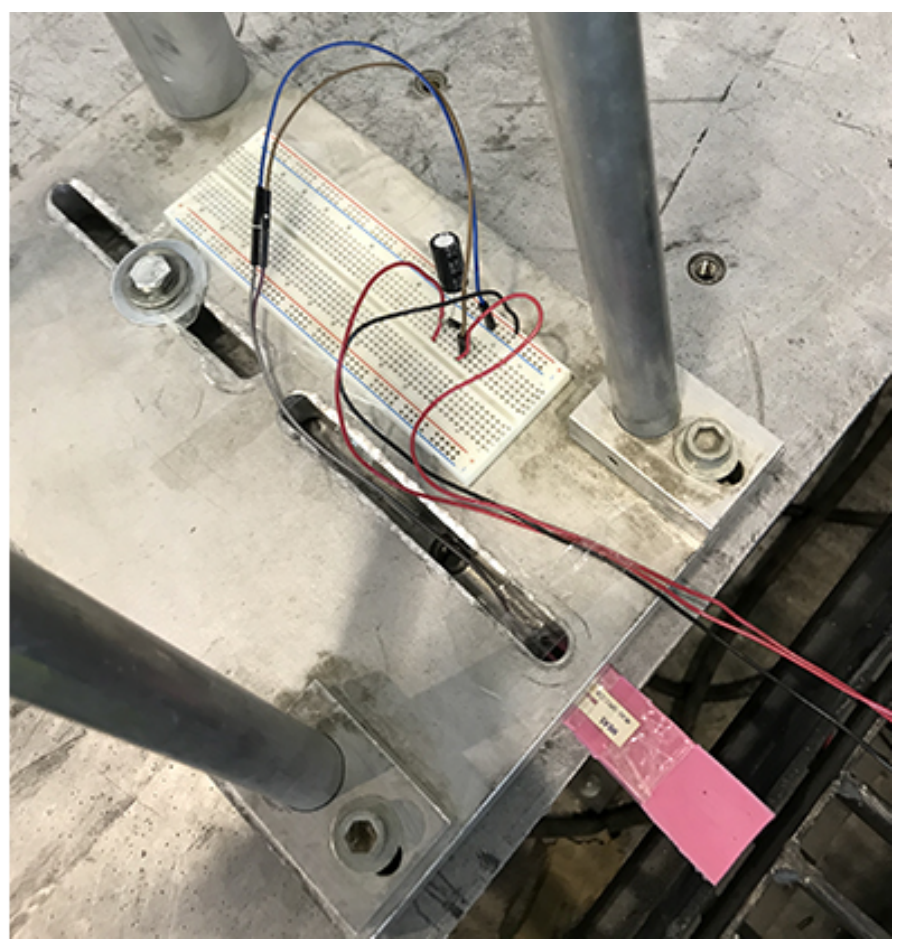

Figure 18. Experimental setup for energy harvesting: rubber plate and vibrating testing machine at the lab. Figure taken from [39].

\subsection{Datalogging Tests-Information in the Cloud}

Tests were carried out to store strain readings in a Google spreadsheet. Data were saved in a comma-separated-values (CSV) file, and the gathered data from the sensor were stored and displayed in the spreadsheet. The process worked as follows: the script read the last saved row with information; then, it received the package of information and provided the needed format to post counter, timestamp, measurement ID, and the raw value in a separate column. At the end of this action, the script increased its counter by 1 to maintain the correct order in the spreadsheet. An example of a test where the sensor reading was sent and stored in a Google spreadsheet is shown in Figure 19.

\begin{tabular}{|r|r|l|l|r|}
\hline & post counter & \multicolumn{1}{|c|}{ timestamp } & measurement ID & raw value \\
\hline \hline 448 & 447 & $01: 06 \mathrm{AM}$ & Strain & 739.00 \\
\hline 449 & 448 & $01: 06 \mathrm{AM}$ & Strain & 740.00 \\
\hline 450 & 449 & $01: 07 \mathrm{AM}$ & Strain & 740.00 \\
\hline 451 & 450 & $01: 07 \mathrm{AM}$ & Strain & 739.00 \\
\hline 452 & 451 & $01: 07 \mathrm{AM}$ & Strain & 740.00 \\
\hline 453 & 452 & $01: 07 \mathrm{AM}$ & Strain & 739.00 \\
\hline 454 & 453 & $01: 07 \mathrm{AM}$ & Strain & 740.00 \\
\hline 455 & 454 & $01: 07 \mathrm{AM}$ & Strain & 739.00 \\
\hline 456 & 455 & $01: 07 \mathrm{AM}$ & Strain & 739.00 \\
\hline 457 & 456 & $01: 07 \mathrm{AM}$ & Strain & 739.00 \\
\hline 458 & 457 & $01: 07 \mathrm{AM}$ & Strain & 740.00 \\
\hline
\end{tabular}

Figure 19. Google spreadsheet with 11 readings (stored in the cloud) coming from the smart strain sensor.

The implemented panel depicted the average strain measured by the smart sensor. During the dashboard-development process, certain tests were carried out to validate the displayed data. Important features of the dashboard and the displayed data during the tests are shown in Figure 20. 


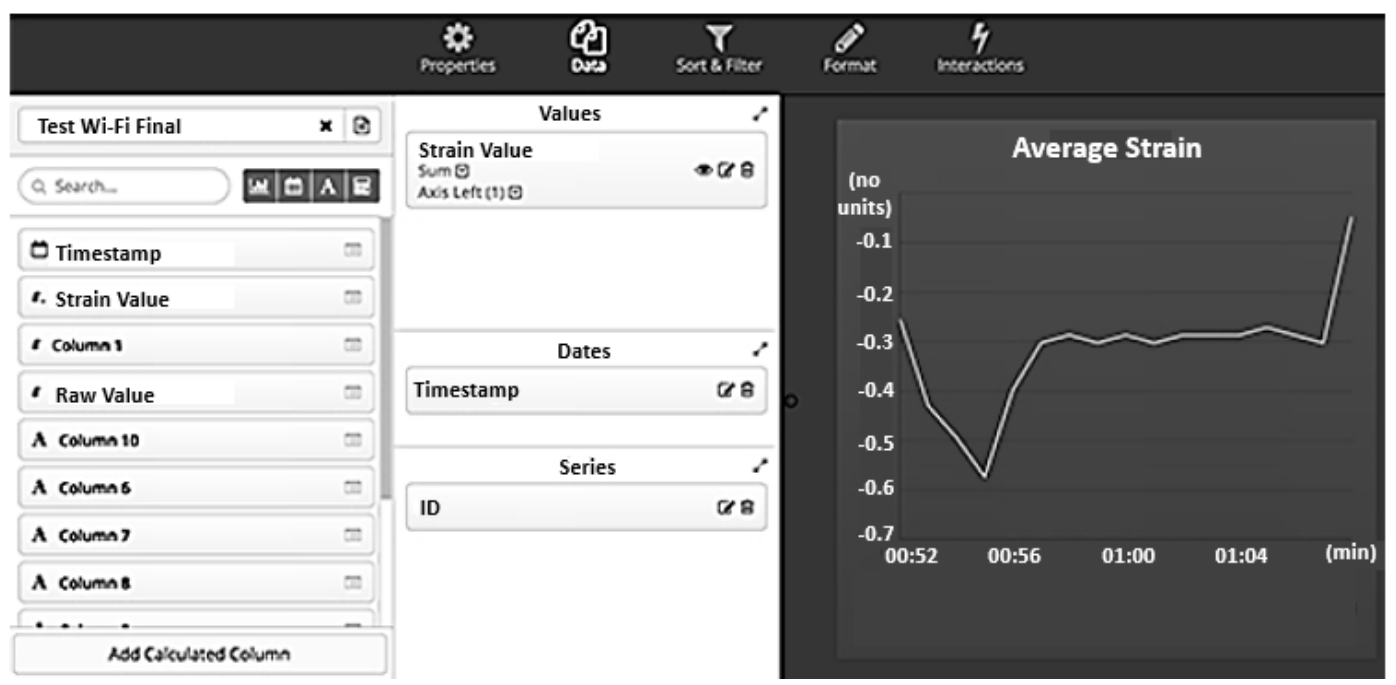

Figure 20. Graph settings for the implemented dashboard. Figure modified from [39].

\section{Results}

This section presents the results of the performed tests on the MRE, the energy-harvesting systems, and the information processed and shared on applications over the Internet.

\subsection{Tensile and Compression Results}

For each of the three designed and manufactured MRE patterns, tensile and compression experiments were performed, and the setup is described in Section 3. For the tensile test, this was carried out for two different instances of the same MRE arrays. Therefore, two results (Replicas 1 and 2) were obtained, as shown in Figure 21.
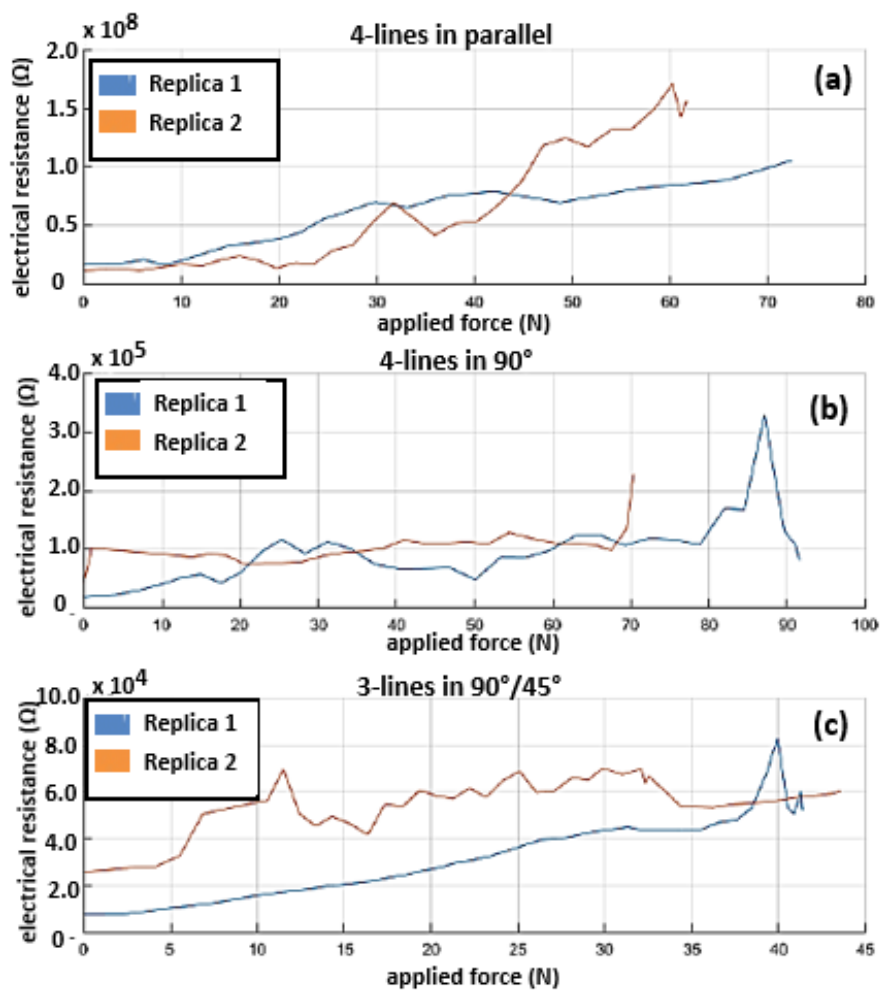

Figure 21. Tensile tests. Electrical resistance vs applied force for three manufactured MRE transducers shown in Figure 3. For each transducer, two tests were carried out (Replicas 1 and 2). (a) Four lines in parallel; (b) four lines in $90^{\circ}$; and (c) three lines in $90^{\circ} / 45^{\circ}$. 
Figure 21 shows that, for the four-parallel-line pattern,the electrical resistance was directly proportional to applied force, indicating the suitability of the MRE for use as a transducer. For the four-line $90^{\circ}$ array, the behavior could not be linearly described because the electrical resistance did not vary in the same proportion as the applied force did. For the three-line $90^{\circ} / 45^{\circ}$ array, Replica 1 demonstrated a quasilinear tendency from 3 to $30 \mathrm{~N}$; however, Replica 2 showed erratic variation as the force increased.

As shown in Figure 22, for the compression tests, the three-line $90^{\circ} / 45^{\circ}$ array with horizontal resistance exhibited quasilinear behavior when the applied force was increased. Both replicas of this array presented this directly proportional dynamic throughout the testing range. For this set of tensile and compression experiments, the MRE array that was most suitable for use as a transducer was the three-line $90^{\circ} / 45^{\circ}$ array with horizontal resistance.

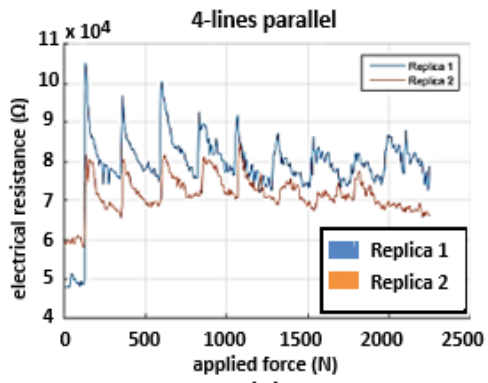

(a)

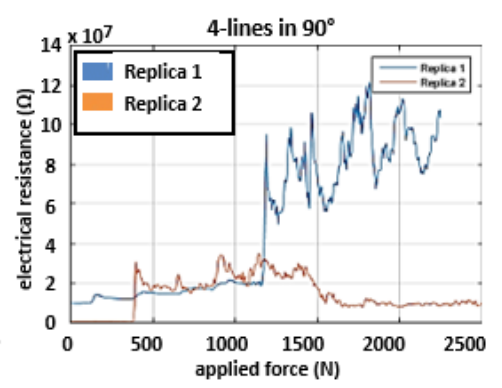

(b)

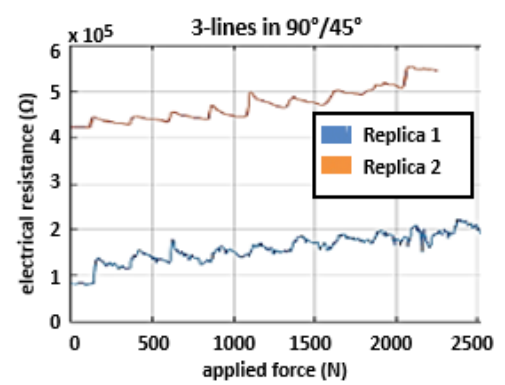

(c)

Figure 22. Compression tests. Electrical resistance vs applied force for three manufactured MRE transducers shown in Figure 3. Two tests (Replicas 1 and 2) were carried out for each transducer.

(a) Four lines in parallel; (b) four lines in $90^{\circ}$; and (c) three lines in $90^{\circ} / 45^{\circ}$.

\subsection{Energy Harvesting-Powering Up Smart Sensor}

For the experimental setup described in Section 4.2, the cantilever beam that included the piezoelectric sensors was tested. The tests started with a cantilever length equal to $5.5 \mathrm{~cm}$ with increments of $0.5 \mathrm{~cm}$. Although the tests were performed with lengths between 5.5 and $8.0 \mathrm{~cm}$, only results between 6.5 and $8.0 \mathrm{~cm}$ are shown here because the highest voltage levels were generated with these lengths.

For each frequency and cantilever length, three experiments were carried out. The justification was to avoid depending on a single result, and checking if there was congruence between the outcomes of the different lengths and frequencies that formed the three replicates of the experiment. The experiment consisted of applying a sinusoidal signal of a certain frequency and taking 500 readings (voltage measurements with a sampling time equal to $0.1 \mathrm{~s}$ ) of the voltage between the piezoelectric-array terminals.

Findings showed that the cantilever length was directly proportional to the generated voltage. Moreover, when the input-signal frequency was between 7.0 and $8.0 \mathrm{~Hz}$, an even greater voltage was produced. The results are shown in Figure 23.

Figure 23 shows that reducing the cantilever length reduced the generated voltage. Moreover, it was detected that, around $7 \mathrm{~Hz}$, the generated voltage had maximal values. Variation was observed in the measured data, and this could have been due to the noise affecting the data-acquisition system.

Due to the length and geometry characteristics of the cantilever beam, it was estimated that there was a resonance frequency around $7 \mathrm{~Hz}$. Furthermore, by increasing the cantilever length, there was a longer lever arm, which contributed to generating a greater piezoelectric reaction. As a consequence, the piezoelectric material underwent a greater degree of deformation and delivered more voltage to the energy-harvesting system. 

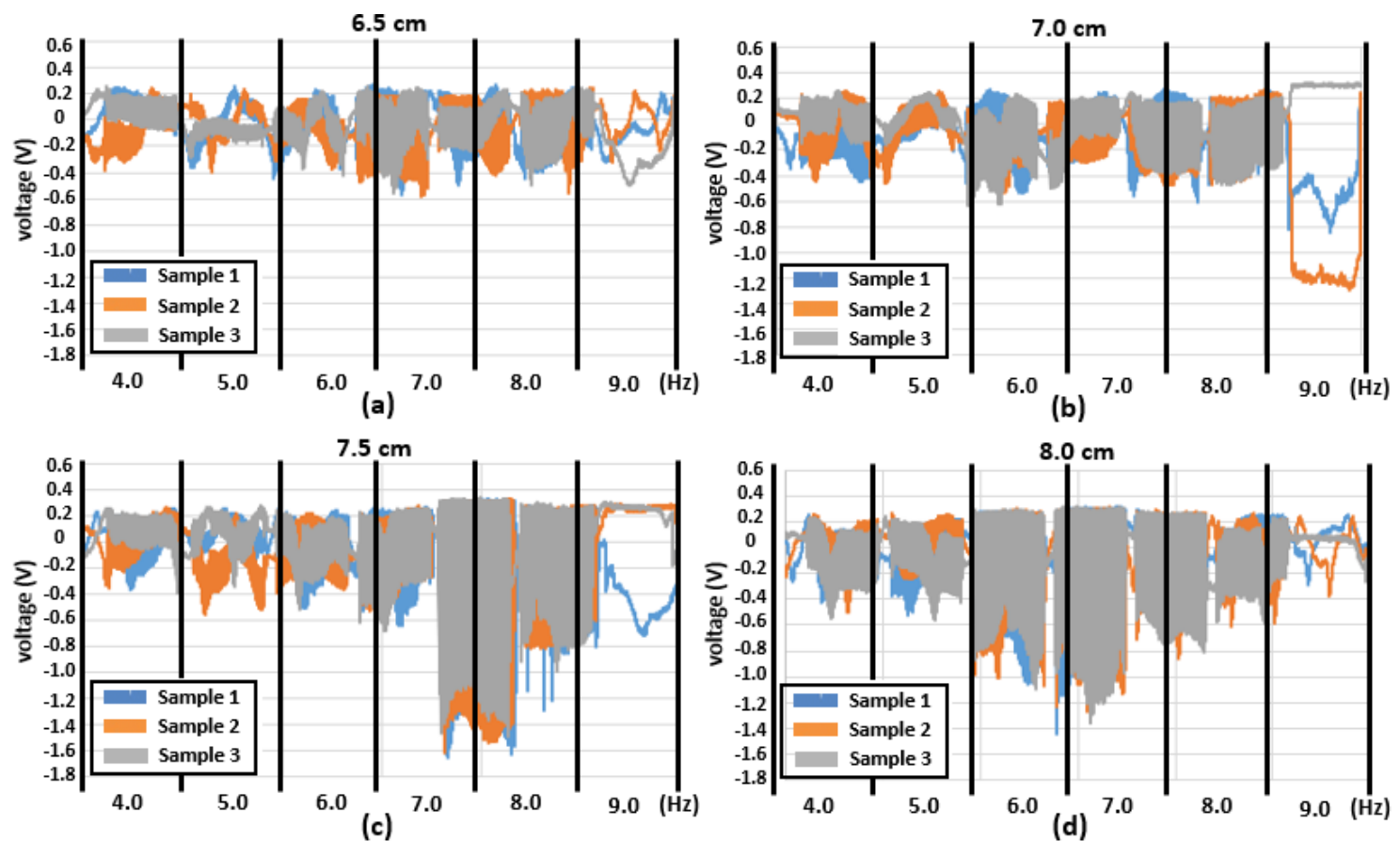

Figure 23. Generated voltage for different cantilever-beam lengths. Three replicas were applied for each test (length and frequency), and 500 measurements were gathered per tested frequency: (a) $6.5 \mathrm{~cm}$; (b) $7.0 \mathrm{~cm}$; (c) $7.5 \mathrm{~cm}$; and (d) $8.0 \mathrm{~cm}$.

For each of the 3 experiments carried out, the cantilever length was set and a signal was applied so that the metallic beam started to vibrate. Considering the length and frequency ranges specified in Section 4.2, for each replica, the results of the series of experiments are depicted in Figures 24-26. Of the three experiments, the maximum generated voltage occurred when the cantilever beam length was $7.5 \mathrm{~cm}$ and the excitation signal had a frequency equal to $8.0 \mathrm{~Hz}$.

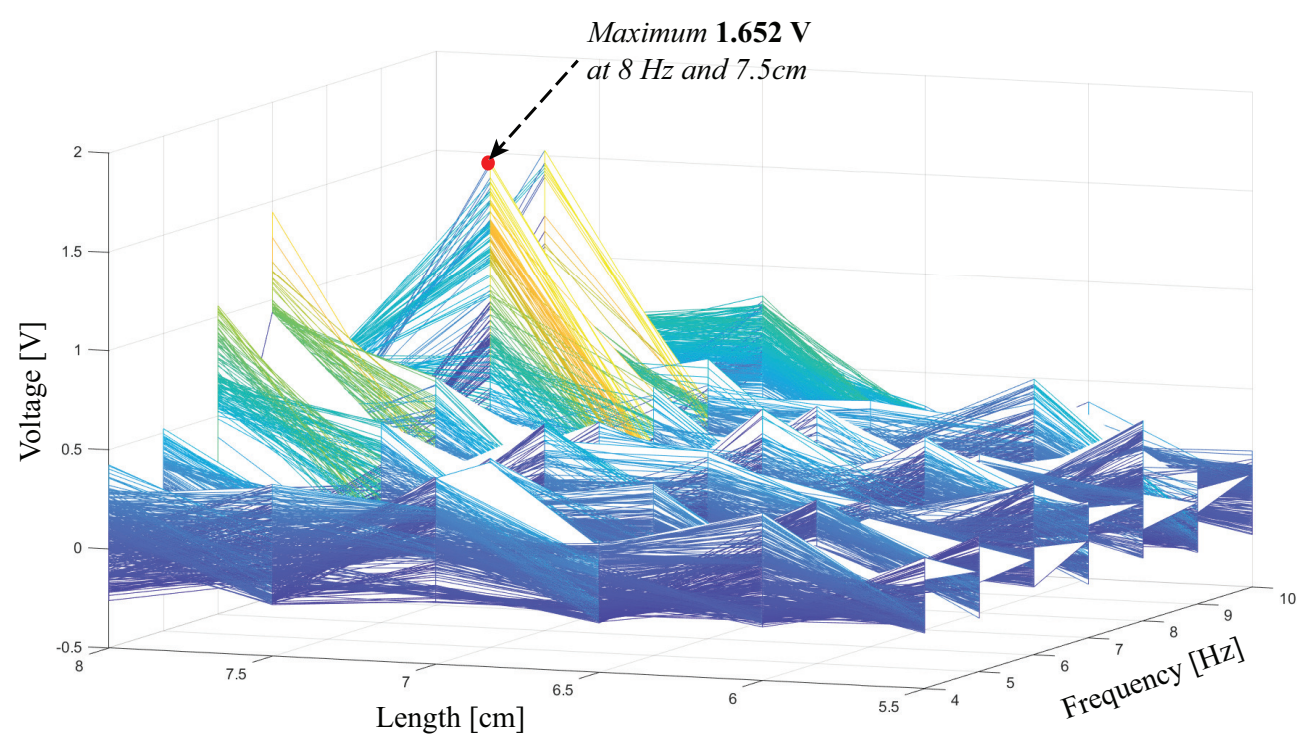

Figure 24. Energy harvesting for Replica 1. The maximum generated voltage was $1.625 \mathrm{~V}$. 


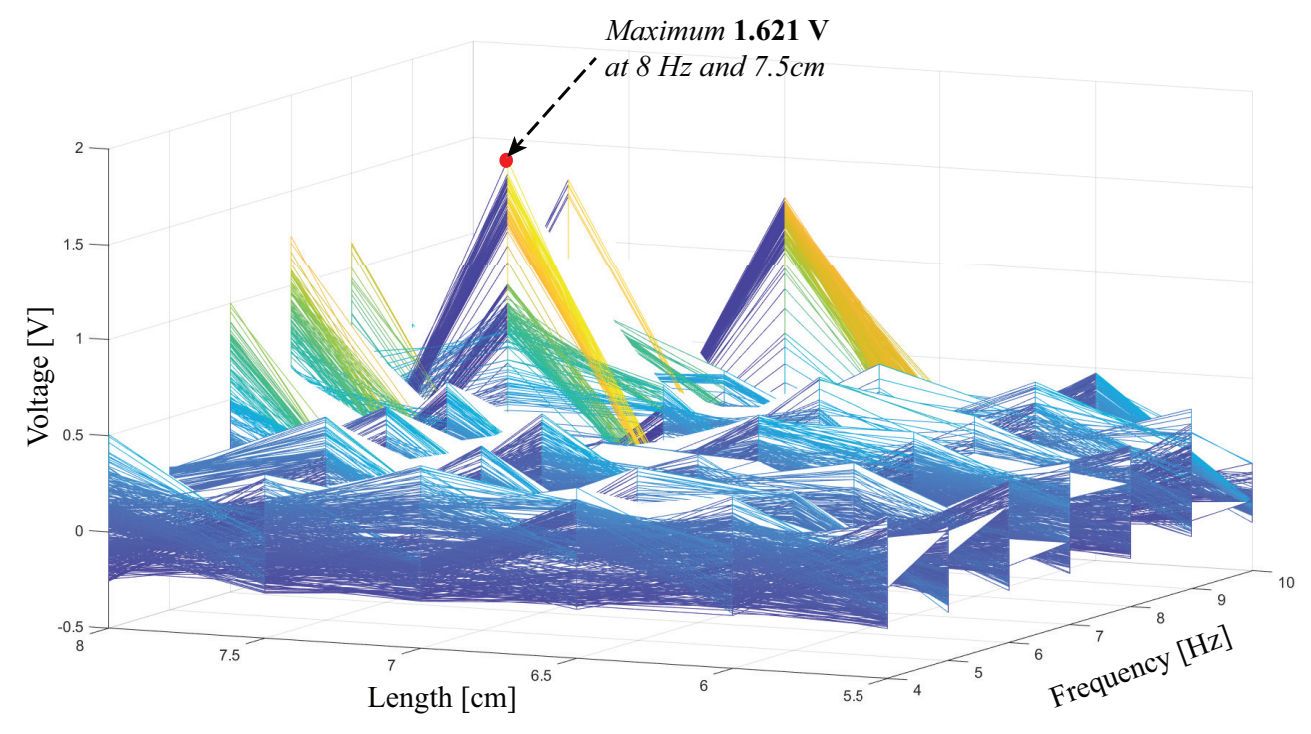

Figure 25. Energy harvesting for Replica 2. The maximum generated voltage was $1.621 \mathrm{~V}$.

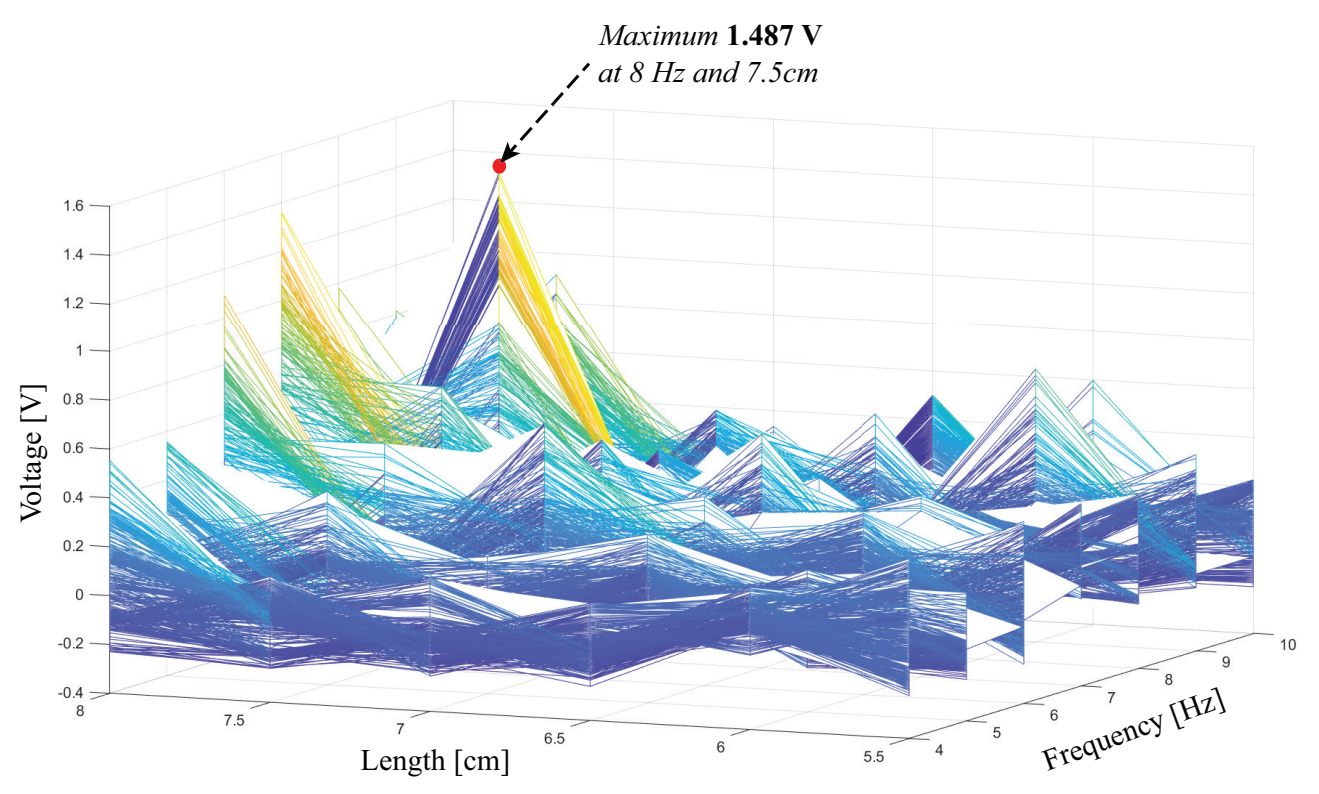

Figure 26. Energy harvesting for Replica 3. The maximum generated voltage was $1.487 \mathrm{~V}$.

The energy harvesting outcomes, which are depicted in Figures 24-26, were concentrated in the 2D graphs shown in Figure 27. The graph of the generated voltage, which is function of the beam length and the input signal frequency, shows the maximum generated voltage with an input signal around $8.0 \mathrm{~Hz}$ and with a length equal to $7.5 \mathrm{~cm}$. For all the tests run, these two combinations of independent variables contributed to harvest the highest amount of energy with values between 1.20 and $1.625 \mathrm{~V}$.

The generated voltage by the cantilever beam went through a diode for rectification, and it was temporarily stored in a capacitor. For the performed tests, the Vout obtained from the best result in Figure 23 is illustrated in Figure 28.

Because the energy-harvesting system was not able to generate sufficient voltage to power up the sensor, and there were only preliminary results for the MRE transducer regarding signal conditioning, the presented outcomes that measured strain inputs and sent them to the dashboard on a website 
were completed on the basis of a strain-gauge sensor with a Wheatstone bridge and a signal amplifier. Moreover, the executed tests for the dashboard considered a powered-up smart sensor with a regular laboratory power supply.

For this experiment, a commercial rosette-array strain gauge arrangement SGD-6/350-RYT21 by Omega Engineering was employed (Figure 29). The strain gauge was used to measure the strain in the area subjected to deformation by the application of force. To successfully install the strain gauges, there was a preparation and installation process, and for this experiment, an installation kit for strain gauges was employed. It is outside the scope of this report to describe the materials and cementing process regarding the strain-gauge transducer.
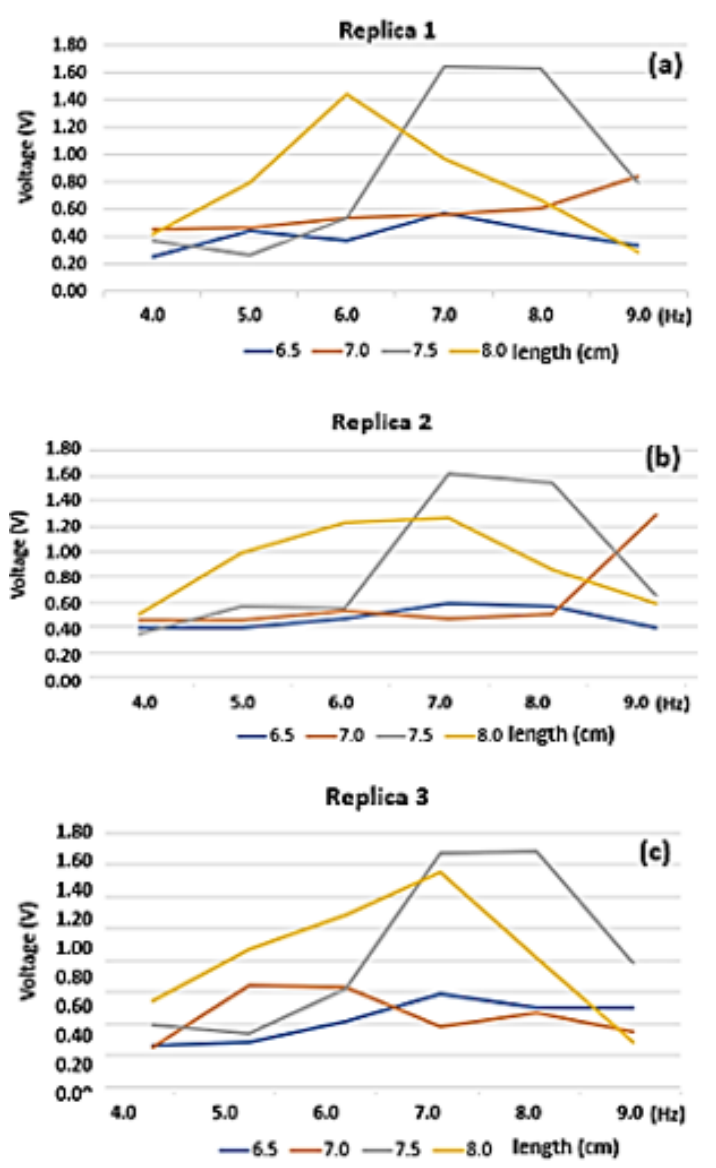

Figure 27. Energy-harvesting tests for the implemented piezoelectric system. (a) Replica 1; (b) Replica 2; and (c) Replica 3.

Due to the nature of strain gauges, signal conditioning is usually required to read the output signal and to send it to the CPU. To solve this problem, two circuits were implemented, a Wheatstone bridge and a signal amplifier with operational amplifiers. As part of the design effort, both circuits were simulated in the Proteus Design Suite to validate their functioning.

A Wheatstone bridge circuit is typically employed to measure the difference in electrical resistance of a strain gauge when a force is applied on the surface where the strain gauge is located. This type of circuit needs a power supply to excite the circuit, and an output voltage signal that is proportional to the applied force is produced. For this research, just one strain gauge of the $0^{\circ} / 45^{\circ} / 90^{\circ}$ three-element strain rosette was used to measure uniaxial stress. The utilized Wheatstone bridge configuration was the quarter-bridge three-wire array. This array with one strain gauge and three resistors with the same electrical resistance value ( $350 \Omega$ in this instance, cancels the thermal effects generated for the lead wires. The second circuit that constituted the signal-conditioning section was a differential amplifier to increase the voltage. The voltage signal coming from the Wheatstone bridge was in the order of 
$\mathrm{mV}$, and it had to be amplified to be read by the microcontroller. The employed operational amplifier was the LM741 integrated circuit powered with -5 and $5 \mathrm{~V}$ to keep the amplified voltage within these limits. After the preliminary tests, the signal-conditioning circuits were placed and welded onto a perforated phenolic plate and inside a protective case designed using computer-aided-design (CAD) software. For more information on Wheatstone bridges and signal conditioning (amplification), refer to $[1,2]$.

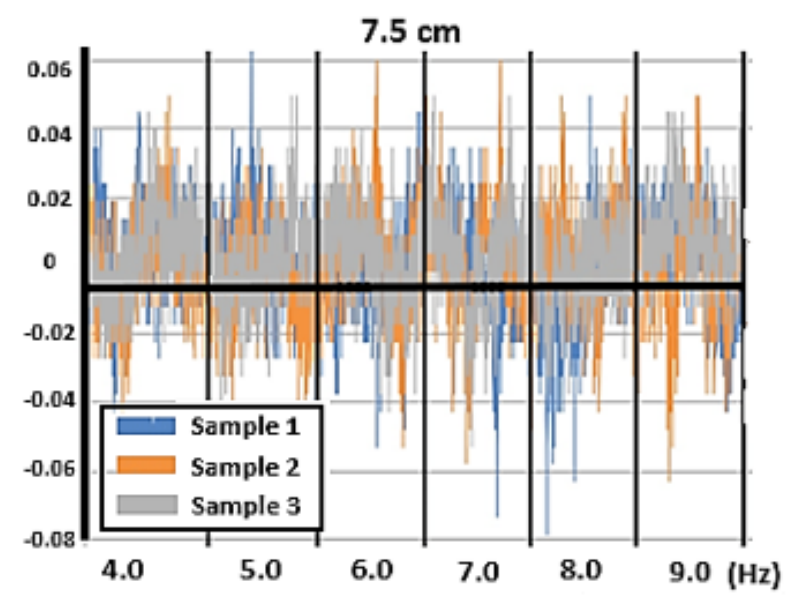

Figure 28. Measured output voltage (Vout) for a $7.5 \mathrm{~cm}$ length cantilever beam with three piezoelectric sensors, as shown in Figure 13. Three samples were applied for each test (length and frequency). Small black numbers, accumulated voltage measurements.

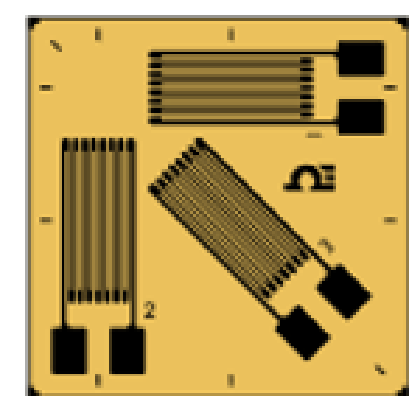

Figure 29. Rectangular rosette $0^{\circ} / 45^{\circ} / 90^{\circ}$ strain-gauge array; model: SGD-6/350-RYT21; $350 \Omega$ of nominal resistance, $16.3 \mathrm{~mm}$ per side with a gauge factor of 2.13 .

\subsection{Dashboard-Towards an IoT approach}

The dashboard was able to show the information obtained by a Google spreadsheet, and the data in the graph were refreshed every minute. The line chart with the average strain over time is depicted in Figure 20.

\section{Discussion}

This study provided important knowledge with regard to the manufacturing process of MRE transducers and the addition of nanoparticles. Experimental tests were performed to cure the MRE layers. Some of them did not work, and acceptable results were obtained when both layers were cured, and the nanoparticles were added in the correct proportion. Furthermore, the mold max 30 silicone rubber with its catalyst worked well for the 3D printing of MRE molds. It was crucial to keep in mind the goal of replacing the strain-gauge transducer with an MRE transducer.

After several tests, the composition of $90 \%$ silicone rubber and $10 \%$ catalyst generated a satisfactory result; on that basis, the nanoparticles were included in the MRE. The initial tests were carried out with iron (II and III) oxide nanopowder, but the relationship between strain and electrical 
resistance was not proportional to allow strain measurement, so it was decided to use multiwall carbon nanotubes with iron, and the experiment delivered satisfactory results. Regarding the ratio of silicone rubber and multiwall carbon particles, the ratio of $97 \%$ silicone rubber (ratio 10:1 of silicone rubber and catalyst) and 3\% MWCNT-Fe particles presented satisfactory electrical-resistance characteristics, as shown in Figures 21 and 22.

On the basis of the MRE transducer results, from the tensile tests in Figure 21, for the four-line parallel lines pattern, the replicas showed similar behavior. At the beginning of the test, a linear tendency was observed, but the slopes of the graphs were different. The rate of resistance change was higher in Replica 2 than that in Replica 1. For the 90-degree array, similarity between the two replicas was observed. The behavior could not be linearly described because, in Replica 2, electrical resistance did not vary as much as that in Replica 1 did. For the 45-degree array, Replica 1 had an almost perfectly linear tendency throughout the whole test. However, Replica 2 showed an increase in resistance as the force went up, but some noise was present from 5 to $35 \mathrm{~N}$.

According to the results of the compression tests shown in Figure 22, a high increase in electrical resistance was instantly observed when the load was applied; then, it declined as the system remained at rest. This behavior was detected for all arrays and with both replicas. Moreover, Replica 1 of the 90-degree array showed the most straightforward relation between applied force and electrical resistance before crossing the $1000 \mathrm{~N}$ border. Electrical resistance technically remained the same when the load was static. For the horizontal resistance of the 45-degree array, Replicas 1 and 2 showed almost equal behavior, although there was an offset between the two responses. A possible justification for this is that the base resistance of each was different, but the increasing steps were similar. In addition, there was some noise in the output resistance when the forces were applied; however, variation was very low and output resistance could be considered as being at a constant value throughout the force-step increase.

The observed results in Figures 21 and 22 were dependent on the hands-on manufacturing of the MRE transducers. The MREs were manually manufactured without following rigorous industrial standards, and this led to variations in the electrical resistance. For example, distribution of the multiwall carbon nanotubes with iron (MWCNT-Fe) following the guidelines in Figure 5 was not the same in all three lines, and this affected the relationship between electrical resistance and applied force. Additionally, by measuring the electrical resistance with a multimeter instead of using specialized electrodes, variations in the readings could have been generated. These peculiarities in MRE construction were part of the behavior of the prototypes.

With regard to the energy-harvesting tests with the piezoelectric sensor merged with a cantilever beam, data in Figure 27 show that the cantilever with a $7.5 \mathrm{~cm}$ length generated the highest voltage levels when the input signal had frequencies of 7 and $8 \mathrm{~Hz}$. Under these conditions, the piezoelectric array was able to generate $1.60 \mathrm{~V}$, which was the highest obtained voltage. The experimental trials included testing with an array of three piezoelectric transducers in parallel (Figure 13), and this configuration allowed for generation of a greater voltage than that generated with a single piezoelectric element. The detected opportunity area was the signal-conditioning circuit with the diode and capacitor. The required voltage to energize both elements was too high compared to the voltage generated by the cantilever, and all the collected energy was lost. Although the voltage signals contained noise, a decrease or increase in voltage was observed for the different experiments, and, owing to that behavior, preliminary conclusions could be generated. Moreover, having generated similar maximum voltage values for the testing conditions contributes to the reproducibility of the experiments.

Even though the complete prototype was tested with a strain-gauge transducer and powered up with a power supply, the results were promising and reinforced two research lines in smart sensors-how to increase the collected energy in a cantilever beam with embedded piezoelectric sensors, and how to reduce the required energy to rectify and retain the gathered voltage for further storage in the smart sensor. 
For the CPU, wireless communication, and IoT capabilities, this study offers a manner of passing the data of the smart-sensor subsystems and data post-processing to cloud computing. For the tests, decisions were made regarding the CPU, and the best option was WEMOS-D1-R1, which was programmed in the familiar Arduino development environment. The ESP8266 Wi-Fi module was the implemented wireless approach, which was compatible with the WEMOS-D1-R1 through the Arduino integrated development environment. Moreover, IoT capabilities included the revision of commercial options to send data to the cloud, store them in Google sheets, and display them on a dashboard developed in ClicData software.

The complete prototype (Figure 15) included the design and manufacture of protective cases and the communication connection among the subsystems. Although these last two activities were not the contribution of this research, they were also important due to the time dedicated to design and to manufacture the complete prototype of the smart sensor.

\section{Conclusions and Future Work}

A low-cost macro-smart-sensor prototype manufactured with commercial technology, connected to the Internet for strain monitoring on metallic surfaces, was designed and fabricated. An alternative approach for well known and applied strain gauges was manufactured, i.e., an MR elastomer, which responded with electrical-resistance variation when force was applied to it. Moreover, the research outcomes included preliminary but relevant results on energy harvesting, and complete tests with a smart sensor that included IoT capabilities.

An energy-harvesting system was explored. The research objective included the design and manufacture of a beam in cantilever-containing piezoelectric sensors fused in a special rubber. The system demonstrated that it was able to generate up to $1.6 \mathrm{~V}$ under the employed testing conditions; however, there was a strong opportunity area regarding voltage rectification and holding.

The benefits of processing data in the cloud and presenting them graphically on the Internet were also shown. The dashboard was able to show the obtained information from the Google spreadsheet, connected to the smart sensor via a wireless protocol through the Internet, and taking only one minute to refresh the data on the dashboard.

Preliminary results showed the potential of this smart sensor for health monitoring in the automotive, aeronautical, and construction industries. For these applications, smart sensors could be embedded in structures, and thus constantly monitor the state of the system and detect potential failure conditions.

The prototype presented areas of opportunity for improvement. Better control to create MR elastomer samples and maintain a uniform number of nanoparticles could be tested. Moreover, the amount and array of nanoparticles could be improved to obtain a more linear and directly proportional relation between MRE strain and electrical resistance. The energy-harvesting system could also have a better design, and different cantilever geometric shapes could be tested to harvest more energy from the environment. Another area of opportunity was detected in the validation experiments, since some measurements were made with a multimeter. This condition could be improved by using embedded electrodes in the cantilever or the MRE to measure electrical resistance or generated voltage following another sensing paradigm.

As future work, a potential research direction is to increase the harvested energy, and improve voltage rectification and holding efficiency. In this context, noise in the voltage measurements offers an opportunity for improvement. A possible solution would be to apply a low-pass filter or to employ electrodes for measuring electrical resistance in the MRE. The latter option was studied with dielectric elastomer sensors that had two electrodes and showed the potential to be applied in SHM and robotics, among others [50].

In addition, the signal-conditioning stage needs to be implemented. An option is to connect electrodes to the MRE and process the signal to make it suitable for the CPU. Other potential work is comparing the smart sensor with other approaches that could be developed (to the best 
of authors' knowledge, there is no reported product with similar attributes to the one we have reported). Additionally, after improving the characteristics and capabilities of the prototype, a digital twin could be generated to complement the monitoring and diagnoses of the system. The experiment-validation results highlighted the potential of the smart-sensor for use in diverse structural-health-monitoring applications.

Author Contributions: Conceptualization and design, J.d.-J.L.-S.; experiment development and validation-test tutoring, J.d.-J.L.-S., J.C.T.-M., and R.-A.R.-M.; state-of-the-art revision update and original-draft writing, L.C.F.-H.; paper writing-revision and editing, A.V.-M. and R.A.R.-M. All authors have read and agreed to the published version of the manuscript.

Funding: Authors are grateful to Tecnologico de Monterrey, through Campus City Research and Technology for funding the publication cost of this research.

Acknowledgments: The authors are grateful to Universidad de Monterrey, through the Center for Innovation in the Design of Packaging, ABRE for allowing the tensile, compression, and energy-harvesting tests to be carried out in its laboratories. Moreover, the authors would like to thank all the undergraduate students for their participation and commitment to carrying out the experiment work of this research. The authors are also grateful to the Metalsa company for its collaboration in carrying out this work.

Conflicts of Interest: The authors declare no conflict of interest.

\section{References}

1. Alciatore, D.G.; Histand, M.B. Sensors. In Mechatronics and Measurement Systems; McGraw-Hill: New York, NY, USA, 2007; pp. 354-361.

2. Doebelin, E.O. Force, Torque, and Shaft Power Measurement. In Measurement Systems: Application and Design; McGraw-Hill Education: London, UK, 2003; pp. 446-451.

3. Perales-Martínez, I.A.; Palacios-Pineda, L.M.; Lozano-Sánchez, L.M.; Martínez-Romero, O.; Puente-Cordova, J.G.; Elías-Zúñiga, A. Enhancement of a magnetorheological PDMS elastomer with carbonyl iron particles. Polym. Test. 2017, 57, 78-86. [CrossRef]

4. Li, W.H.; Zhang, X.Z.; Du, H. Magnetorheological elastomers and their applications. In Advances in Elastomers I: Blends and Interpenetrating Networks; Visakh, P.M., Thomas, S., Chandra, A.K., Mathew, A.P., Eds.; Springer: Berlin, Germany, 2013; pp. 357-374.

5. Liu, T.; Xu, Y. Magnetorheological Elastomers: Materials and Applications. In Smart and Functional Soft Materials; Dong, X., Ed.; IntechOpen: Berlin, Germany, 2019; pp. 3-23.

6. Kumbhar, S.R.; Maji, S.; Kumar, B. Dynamic mechanical analysis of Magnetorheological Elastomer. In Proceedings of the 2013 International Conference on Energy Efficient Technologies for Sustainability, Nagercoil, India, 10-12 April 2013; pp. 870-873.

7. Li, Y.; Li, J.; Li, W.; Du, H. A state-of-the-art review on magnetorheological elastomer devices. Smart Mater. Struct. 2014, 23, 1-24. [CrossRef]

8. Beeby, S.P.; Tudor, M.J.; White, N.M. Energy harvesting vibration sources for microsystems applications. Meas. Sci. Technol. 2006, 17, 1455-1556. [CrossRef]

9. Nayan, H. Power generation using piezoelectric material. J. Mater. Sci. Eng. 2015, 4, 1-4.

10. Lopes, C.M.A.; Gallo, C.A. A review of piezoelectrical energy harvesting and applications. In Proceedings of the 2014 IEEE 23rd International Symposium on Industrial Electronics (ISIE), Istanbul, Turkey, 1-4 June 2014; pp. 1284-1288.

11. Zheng, J.C.; Ruan, T.; Zhu, M.; Marise Bafleur, M.; Dilhac, J.-M. Single Piezoelectric Transducer as Strain Sensor and Energy Harvester Using Time-Multiplexing Operation. IEEE Trans. Ind. Electr. 2017, 64, 9646-9656.

12. Shirvanimoghaddam, M.; Shirvanimoghaddam, K.; Abolhasani, M.M.; Farhangi, M.; Barsari, V.Z.; Liu, H.; Dohler, M.; Naebe, M. Towards a Green and Self-Powered Internet of Things Using Piezoelectric Energy Harvesting. IEEE Access 2019, 7, 94533-94556. [CrossRef]

13. Mo, C.; Davidson, J. Energy harvesting technologies for structural health monitoring applications. In Proceedings of the 2013 1st IEEE Conference on Technologies for Sustainability (SusTech), Portland, OR, USA, 1-2 August 2013; pp. 192-198.

14. Dahkar, L. Overview of Energy Harvesting Technologies. In Triboelectric Devices for Power Generation and Self-Powered Sensing Applications; Springer: Singapore, 2017; pp. 9-34. 
15. Silva, A.F.; Mendes, P.M.; Correia, J.H.; Goncalves, F.; Ferreira, L.A.; Araujo, F.M. Inner car smart flooring for monitoring chassis deformation. In Proceedings of the IEEE Sensors, Christchurch, New Zealand, 25-28 October 2009; pp. 1584-1587.

16. Perelli, A.; Caione, C.; De Marchi, L.; Brunelli, D.; Marzani, A.; Benini, L. Design of an ultra-low power device for aircraft structural health monitoring. In Proceedings of the 2013 Design, Automation \& Test in Europe Conference \& Exhibition (DATE), Grenoble, France, 18-22 March 2013; pp. 1127-1130.

17. Liu, F.; Xiao, L.; Peng, J.; Yang, X. Visual monitoring research of airfoil structural based on virtual test technology. In Proceedings of the 2015 IEEE International Conference on Mechatronics and Automation (ICMA), Beijing, China, 2-5 August 2015; pp. 600-605.

18. Leong, Z.; Al-Taher, A.; Chan, L.; Walters, N.; McGahan, M.; Hayes, S.; Lupu, N.; Murgulescu, I.; Morley, N. Structural Health Monitoring using Magnetostrictive Sensors. In Proceedings of the 2017 IEEE International Magnetics Conference (INTERMAG), Dublin, Ireland, 24-28 April 2017; pp. 1-5.

19. Dong, T.; Kim, N.H. Cost-Effectiveness of Structural Health Monitoring in Fuselage Maintenance of the Civil Aviation Industry. Aerospace 2018, 5, 87. [CrossRef]

20. Kwon, S.H.; Lee, J.H.; Choi, H.J. Magnetic Particle Filled Elastomeric Hybrid Composites and Their Magnetorheological Response. Materials 2018, 11, 1040. [CrossRef] [PubMed]

21. Aziz, S.A.A.; Mazlan, S.A.; Ismail, N.I.N.; Choi, S.-B.; Ubaidillah; Yunus, N.A.B. An enhancement of mechanical and rheological properties of magnetorheological elastomer with multiwall carbon nanotubes. J. Intell. Mater. Syst. Struct. 2017, 28, 3127-3138. [CrossRef]

22. Liu, H.; Zhong, J.; Lee, C.; Lee, S.-W.; Lin, L. A comprehensive review on piezoelectric energy harvesting technology: Materials, mechanisms, and applications. Appl. Phys. Rev. 2018, 5, 041306. [CrossRef]

23. Li, H.; Tian, C.; Deng, Z.D. Energy harvesting from low frequency applications using piezoelectric materials. Appl. Phys. Rev., 2014, 1, 041301. [CrossRef]

24. Safaei, M.; Sodano, H.A.; Anton, S.R. A review of energy harvesting using piezoelectric materials: State-of-the-art a decade later (2008-2018). Smart Mater. Struct. 2019, 28, 113001. [CrossRef]

25. Pozo, B.; Garate, J.I.; Araujo, J.Á.; Ferreiro, S. Energy Harvesting Technologies and Equivalent Electronic Structural Models. Electronics 2019, 8, 486. [CrossRef]

26. Kim, H.S.; Kim, J.H.; Kim, J. A review of piezoelectric energy harvesting based on vibration. Int. J. Precis. Eng. Manuf. 2011, 12, 1129-1141. [CrossRef]

27. Chen, Z.S.; Yang, Y.M.; Deng, G.Q. Analytical and experimental study on vibration energy harvesting behaviors of piezoelectric cantilevers with different geometries. In Proceedings of the 2009 International Conference on Sustainable Power Generation and Supply, Nanjing, China, 6-7 April 2009; pp. 1-6.

28. Uddin, M.N.; Islam, M.S.; Sampe, J.; Wahab, S.A.; Ali, S.H.M. Vibration based T-shaped piezoelectric cantilever beam design using finite element method for energy harvesting devices. In Proceedings of the 2016 IEEE International Conference on Semiconductor Electronics (ICSE), Kuala Lumpur, Malaysia, 17-19 August 2016; pp. 137-140.

29. Kuwano, H.; Van Minh, L.; Nguyen, H.H.; Asanuma, H.; Oguchi, H. Vibration-driven micro energy harvesting with piezoelectric materials. In Proceedings of the 2018 IEEE/ASME International Conference on Advanced Intelligent Mechatronics (AIM), Auckland, New Zealand, 9-12 July 2018; pp. 395-400.

30. Reddy, A.R.; Umaphaty, M.; Ezhilarasi, D.; Gandhi, U. Improved energy harvesting from vibration by introducing cavity in a cantilever beam. J. Vib. Control 2016, 22, 3057-3066. [CrossRef]

31. Patel, R.; Tanaka, Y.; McWilliam, S.; Mutsuda, H.; Popov, A.A. Simply-supported multi-layered beams for energy harvesting. J. Intell. Mater. Syst. Struct. 2017, 28, 740-759. [CrossRef]

32. Xu, J.; Tang, J. Modeling and analysis of piezoelectric cantilever-pendulum system for multi-directional energy harvesting. J. Intell. Mater. Syst. Struct. 2017, 28, 323-338. [CrossRef]

33. Gebresenbet, G.; Aradom, S.; Bulitta, F.S.; Hjerpe, E. Vibration levels and frequencies on vehicle and animals during transport. Biosyst. Eng. 2011, 110, 10-19. [CrossRef]

34. Yan, S.; Ma, H.; Li, P.; Song, G.; Wu, J. Development and Application of a Structural Health Monitoring System Based on Wireless Smart Aggregates. Sensors 2017, 17, 1641. [CrossRef]

35. Rekha, K.S.; Sreenivas, T.H.; Kulkarni, A.D. Remote Monitoring and Reconfiguration of Environment and Structural Health Using Wireless Sensor Networks. J. Intell. Mater. Syst. Struct. 2018, 5, 1169-1175. [CrossRef] 
36. Alonso, L.; Barbarán, J.; Chen, J.; Díaz, M.; Llopis, L.; Rubio, B. Middleware and communication technologies for structural health monitoring of critical infrastructures: A survey. Comput. Stand. Interfaces 2017, 56, 83-100. [CrossRef]

37. Fu, Y.; Mechitov, K.; Hoang, T.; Kim, J.R.; Lee, D.H.; Spencer, B.F., Jr. Development and full-scale validation of high-fidelity data acquisition on a next-generation wireless smart sensor platform. Adv. Struct. Eng. 2019, 22, 3512-3533. [CrossRef]

38. Masoud, M.; Jaradat, Y.; Manasrah, A.; Jannoud, I. Sensors of Smart Devices in the Internet of Everything (IoE) Era: Big Opportunities and Massive Doubts. J. Sens. 2019, 2019, 1-26. [CrossRef]

39. Lozoya-Santos, J.d.-J.; Tudón-Martínez, J.C.; Vargas Martinez, A.; Morales Menendez, R.; Ramírez-Mendoza, R.A. A smart sensor for the measurements of strain and vibrations: A work in progress. In Proceedings of the SPIE 10967: Active and Passive Smart Structures and Integrated Systems XIII 109672B, Denver, CO, USA, 21 March 2019; pp. 1-7.

40. De Lozoya-Santos, J.-J.; Vargas Martinez, A.; Tudón-Martínez, J.C.; Morales Menendez, R.; Ramírez-Mendoza, R.A.; Roman-Flores, A. Smart elastomers for the sensing of force and vibration: A proof of Concept. In Proceedings of the 30th International Conference on Adaptive Structures and Technologies (ICAST2019), Montreal, QC, Canada, 7-11 October 2019.

41. Yarra, S.; Gordaninejad, F.; Behrooz, M.; Pekcan, G. Performance of natural rubber and silicone-based magnetorheological elastomers under large-strain combined axial and shear loading. J. Intell. Mater. Syst. Struct. 2018, 30, 1-15. [CrossRef]

42. Rezaie, E.; Hajalilou, A.; Rezanezhad, A.; Abouzari-Lotf, E.; Arsalani, N. Magnetorheological studies of polymer nanocomposites. In Rheology of Polymer Blends and Nanocomposites: Theory, Modelling and Applications; Sabu, T., Sarathchandran C., Nithin C., Eds.; Elsevier: Amsterdam, The Netherlands, 2020; pp. 263-294.

43. CTR Scientific. Monterrey, Nuevo León, México. Available online: www.ctr.com.mx (accessed on 10 January 2020).

44. Castagnetti, D. Comparison Between a Wideband Fractal-Inspired and a Traditional Multicantilever Piezoelectric Energy Converter. J. Vib. Acoust. 2014, 137, 1-7. [CrossRef]

45. Chen, C.H.; Chen, H.C.; Huang, Y.S.; Hsieh, P.H.; Wu, P.H.; Shu, Y.C. A series-SSHI-Phi interface circuit for piezoelectric energy harvesting with 163\% improvement in extracted power at off-resonance. In Proceedings of the 2016 IEEE Asian Solid-State Circuits Conference (A-SSCC), Toyama, Japan, 7-9 November 2016; pp. 29-32.

46. Chen, B. Introduction to energy harvesting transducers and their power conditioning circuits Low-Power Analog Techniques. In Sensors for Mobile Devices, and Energy Efficient Amplifiers; Springer: Cham, Switzerland, 2019; pp. 3-12.

47. Norouzi, M.; Gilani, M.; Sajjadi Alehashem, S.M.; Vatandoost, H. Dynamic Characterization and Modeling of Isotropic Magnetorheological Elastomers Under Tensile-Compressive Loadings. IEEE Trans. Magnet. 2017, 53, 1-12. [CrossRef]

48. Kukla, M.; Górecki, J.; Malujda, I.; Talaska, K.; Tarkowski, P. The Determination of Mechanical Properties of Magnetorheological Elastomers (MREs). Procedia Eng. 2017, 177, 324-330. [CrossRef]

49. Schubert, G.; Harrison, P. Large-strain behavior of Magneto-Rheological Elastomers tested under uniaxial compression and tension, and pure shear deformations. Polym. Test. 2015, 42, 122-134. [CrossRef]

50. Ni, N.; Zhang, L. Dielectric Elastomer Sensors. In Elastomers; Çankaya, N., Ed.; IntechOpen: Rijeka, Croatia, 2017; pp. 231-253.

(C) 2020 by the authors. Licensee MDPI, Basel, Switzerland. This article is an open access article distributed under the terms and conditions of the Creative Commons Attribution (CC BY) license (http://creativecommons.org/licenses/by/4.0/). 